\title{
TITLE:
}

\section{Classification and characteristics of Floquet factorisations in linear continuous-time periodic systems}

\author{
AUTHOR(S): \\ Zhou, Jun
}

\section{CITATION:}

Zhou, Jun. Classification and characteristics of Floquet factorisations in linear continuous-time periodic systems. International Journal of Control 2008, 81(11): 16821698

\section{ISSUE DATE:}

2008-11

URL:

http://hdl.handle.net/2433/85332

\section{RIGHT:}

This is an electronic version of an article published in International Journal of Control Vol.81 No.11 pp., 1682-1698, 2008. International Journal of Control is available online at: http:/ /www.informaworld.com; 許諾条件により本文は2009-12 01に公開; This is not the published version. Please cite only the published version.; この論文は出版社版でありません。 引用の際には出版社版をご確認ご利用ください。 


\title{
Classification and Characteristics of Floquet Factorizations in Linear Continuous-Time Periodic Systems
}

\author{
Jun $\mathrm{ZHOU}^{*}$ \\ Department of Electrical Engineering, Kyoto University
}

\begin{abstract}
This paper has two purposes. Firstly, the paper is devoted to collecting basic facts about the Floquet theorem and various Floquet factorization algorithms claimed for finite-dimensional linear continuous-time periodic (FDLCP) systems. Secondly, the paper presents a unified representation framework for various Floquet factorizations in FDLCP systems, while structural and analytic characteristics about them are examined as well. More precisely, the following aspects are considered: (i) algorithms for Floquet factorizations; (ii) characteristics of Floquet factors; (iii) relationships and properties among Floquet factorizations. Most results are reported for the first time, while the others are generalized versions of existing ones.
\end{abstract}

Key words: martrix logarithm, Floquet factorization, simplicity, reducibility, commutativity

\section{Introduction}

The Floquet theorem, or more generally Floquet theory, can be traced back to 1883 [9], which presents us Floquet factorizations for state transition matrices, fundamental matrices and solutions to periodic differential equations. Perodic differential equations frequently appear in control and system applications related to finite-dimensional linear continuous-time periodic (FDLCP) modelings $[8,12,19,28]$. Typical problems include stabilization of helicopter rotors and ships in waves, and reduction of electro-mechanical oscillations or swing in electricity generators $[1,6,8,11,24,25]$. As a matter of fact, the Floquet theorem is originally developed to transfer periodic differential equations into ones with constant coefficients and has been one of the kernel results for analysis and synthesis in FDLCP control systems, without which some important developments in the FDLCP field may not be attainable. For example, asymptotic stability in FDLCP systems can be better dealt with if Floquet factorizations are available [31, 33]; control can be implemented via real Floquet factorizations [22]; harmonic controllability criteria are established also with Floquet factorizations [34]; last but not least, frequency-domain aspects about FDLCP systems can be examined by means of Floquet factorizations $[7,16,30,32,33]$. It is worth mentioning that Floquet theory has been extended in partial differential equations [16] as well.

With such a long history of Floquet theory, one probably feels that the Floquet theorem has been completely examined and perfectly developed. To one's surprise, if one scans through the

\footnotetext{
${ }^{*}$ Kyotodaigaku-Katsura, Nishikyo-ku, Kyoto 615-8510, Japan, Tel: +81-75-3832260, Fax: +81-75-3832260, Email: zhouj@kuee.kyoto-u.ac.jp
} 
literature, one will quickly find that this is not the case. On the one hand, existence of Floquet factorizations has been dealt with in numerous textbooks on differential equations $[1,8,14,23]$ through various matrix logarithm algorithms, and basic properties of Floquet factorizations are also reported $[8,19]$. On the other hand, how to interpret and compare the Floquet factorizations obtained via different matrix logarithm algorithms remain as open problems. In addition, some results were interpreted somehow inappropriately to some degree.

Outline. Section 2 collects notation and terminologies about matrix logarithm algorithms. Section 3 discusses existence and classification of Floquet factorizations. Lemmas and theorems in Sections 2 and 3 are restatements or generalizations of existing results about Floquet theory. Properties of Floquet factors are examined in Section 4, while those about Floquet factorizations as a whole are scrutinized in Section 5. Most theorems in Sections 4 and 5 are reported for the first time. Conclusions are given in Section 6. $\mathcal{R}$ and $\mathcal{C}$ are the set of all real numbers and that of all complex numbers, respectively. $\mathcal{Z}$ is the ring of all integers. $\mathcal{F}$ represents either $\mathcal{R}$ or $\mathcal{C} . \lambda(\cdot)$ is the set of eigenvalues of a matrix (.). Let $J$ be a nonempty open interval in time.

\section{Preliminaries to Matrix Logarithm Algorithms}

First we review the (scalar) complex logarithm. Let $\zeta \neq 0 \in \mathcal{C}$. We have by complex analysis [15, $18,29]$ that the $p$-th logarithm branch of $\zeta$ is given by

$$
\ln (p, \zeta)=\operatorname{Ln}(|\zeta|)+j(\arg (\zeta)+2 \pi p), \quad p \in \mathcal{Z}
$$

where $\operatorname{Ln}(\cdot)$ means the logarithm of a positive real number $(\cdot)$ and $\arg (\cdot)$ is the principal argument with $-\pi<\arg (\cdot) \leq \pi$. By (1), there are infinitely many branches in a complex logarithm, which are distributed on the Riemann surface consisting of infinitely many ramifications. Conventionally, the branch with $p=0$ is called the principal complex logarithm of $\zeta$ and written as

$$
\ln (\zeta)=: \ln (0, \zeta)=\operatorname{Ln}(|\zeta|)+j \arg (\zeta)
$$

In the sequel we collects lemmas about matrix logarithm algorithms. By the author's best understanding, there are some inappropriate arguments in the proofs for these lemmas in textbooks on ordinary differential equations [4],[8],[13],[19]. To clarify those questionable arguments, we will include detailed proofs whenever necessary.

\subsection{Matrix Logarithm by the Cauchy Integration Formula [15, 19]}

Let $C$ be a $n \times n$ matrix and $I$ be the identity of dimension $n \times n$. We write

$$
B=\frac{1}{2 \pi j} \oint_{\partial \Omega} \ln (p, z)[z I-C]^{-1} d z, \quad p \in \mathcal{Z}
$$

The integral (3) is well-defined by the Cauchy integration formula [29, p.53], provided that a $p$-th logarithm branch and an integral path $\partial \Omega$ are specified properly. More precisely, $\oint_{\partial \Omega}$ denotes a line integration along $\partial \Omega$, which is the boundary of $\Omega \subset \mathcal{C} \backslash\{0\}$ and consists of a finitely many simple closed curves that do not intersect themselves and orient in the positive direction. $\Omega$ is an open set containing all eigenvalues of the constant matrix $C$.

Lemma 1 If $C \in \mathcal{F}^{n \times n}$ is nonsingular, the Cauchy integration formula (3) satisfies $C=e^{B}$. Furthermore, if $C$ is real, then $B \bar{B}=\bar{B} B$ and $e^{B+\bar{B}}=C^{2}$. Here $(\cdot \bar{\cdot})$ denotes the conjugate of $(\cdot)$. 
Proof of Lemma 1 We refer the reader to Theorem 7.5.1, Lemma 8.1.1, Lemma 8.1.2 and Theorem 8.1.3 of [19] for all the assertions.

Lemma 2 Assume in (3) that $\ln (p, z)$ is the principal branch, i.e., $p=0$. If $C \in \mathcal{R}^{n \times n}$ is nonsingular without negative eigenvalues, then $B$ is real and satisfies $C=e^{B}$.

Proof of Lemma 2 By the assumption on $C$, an open set $\Omega$ exists such that (3) is well-defined. Furthermore, $\ln (p, z)$ is analytic on $\Omega$, and $[z I-C]^{-1}$ is a real meromorphic function on $\Omega$ that is analytic on $\Omega \backslash \lambda(C)$. The theorem of residue [18, 29] and (3) lead that

$$
\begin{aligned}
B & =(2 \pi j)^{-1}\left[2 \pi j \sum_{i=1}^{n} \operatorname{Res}_{z=\lambda_{i}}\left\{\ln (p, z)[z I-C]^{-1}\right\}\right] \\
& =\sum_{i=1}^{n} \operatorname{Res}_{z=\lambda_{i}}\left\{\ln (p, z)[z I-C]^{-1}\right\}
\end{aligned}
$$

where $\operatorname{Res}_{z=\lambda_{i}}(\cdot)$ is the residue of $(\cdot)$ at $z=\lambda_{i}$. These residues are either real or pairs of conjugate numbers since $C$ is real and $\ln (p, z)$ takes the principal branch. We conclude that $B$ is real.

\subsection{Matrix Logarithm by Jordan Canonical Forms [4, 13, 14]}

For $C \in \mathcal{F}^{n \times n}$, choose a $n \times n$ nonsingular matrix $T$ such that $C=T^{-1} \operatorname{diag}\left[J_{1}, \cdots, J_{\alpha}\right] T$ with $J_{i}$ being a $n_{i} \times n_{i}$ Jordan block corresponding to an eigenvalue $\lambda_{i}$ of $C$, that is

$$
J_{i}=\lambda_{i} I_{i}+E_{i} \in \mathcal{F}^{n_{i} \times n_{i}}, \quad i=1,2, \cdots, \alpha, \quad \sum_{i} n_{i}=n
$$

where $I_{i}$ is the $n_{i} \times n_{i}$ identity matrix and $E_{i}$ is the following $n_{i} \times n_{i}$ nilpotent

$$
E_{i}=\left[\begin{array}{cccc}
0 & 1 & \cdots & 0 \\
\vdots & \ddots & \ddots & \vdots \\
0 & \ddots & \ddots & 1 \\
0 & 0 & \cdots & 0
\end{array}\right]
$$

Hence, $E_{i}^{l}=0$ for all $l \geq m_{i}$ where $m_{i} \geq 0$ is an integer satisfying $m_{i}<n_{i}$.

Based on the above notation, we can define the following formula for $B$ with $C \in \mathcal{F}^{n \times n}$.

$$
B=T^{-1} \operatorname{diag}\left[\ln \left(p_{1}, \lambda_{1}\right) I_{1}-\sum_{i=1}^{m_{1}-1} \frac{\left(-E_{1}\right)^{i}}{i \lambda_{1}^{i}}, \cdots, \quad \ln \left(p_{\alpha}, \lambda_{\alpha}\right) I_{\alpha}-\sum_{i=1}^{m_{\alpha}-1} \frac{\left(-E_{\alpha}\right)^{i}}{i \lambda_{\alpha}^{i}}\right] T
$$

where $p_{1}, \cdots, p_{\alpha} \in \mathcal{Z}$. If $C$ is real, there exists a real and nonsingular matrix $T$ [4, pp.106-107] such that $C=T^{-1} \operatorname{diag}\left[R_{1}, \cdots, R_{\beta}, J_{\beta+1}, \cdots, J_{\alpha}\right] T$ with $J_{i}$ being a $n_{i} \times n_{i}$ Jordan block corresponding to a real eigenvalue $\lambda_{i}$ as described in the above, while $R_{i}$ is a real Jordan block corresponding to a complex eigenvalue $\lambda_{i}=r_{i}+j g_{i}$; that is, for each $i=1,2, \cdots, \alpha$

$$
\begin{aligned}
R_{i} & =\Lambda_{i}+F_{i} \in \mathcal{R}^{n_{i} \times n_{i}} \\
& =\left[\begin{array}{cccc}
S_{i} & 0_{2} & \cdots & 0_{2} \\
\vdots & \ddots & \ddots & \vdots \\
0_{2} & \ddots & \ddots & 0_{2} \\
0_{2} & 0_{2} & \cdots & S_{i}
\end{array}\right]+\left[\begin{array}{cccc}
0_{2} & I_{2} & \cdots & 0_{2} \\
\vdots & \ddots & \ddots & \vdots \\
0_{2} & \ddots & \ddots & I_{2} \\
0_{2} & 0_{2} & \cdots & 0_{2}
\end{array}\right], \quad S_{i}=\left[\begin{array}{cc}
r_{i} & -g_{i} \\
g_{i} & r_{i}
\end{array}\right]
\end{aligned}
$$

and $0_{2}$ is the $2 \times 2$ zero matrix, $I_{2}$ is the $2 \times 2$ identity matrix. The definitions for $\Lambda_{i}$ and $F_{i}$ are obvious, and thus $F_{i}$ is also a nilpotent matrix. 
Accordingly we define the following formula for $B$ with $C$ being real.

$$
\begin{array}{r}
B=T^{-1} \operatorname{diag}\left[\ln \left(p_{1}, \Lambda_{1}\right)-\sum_{i=1}^{m_{1}-1} \frac{1}{i}\left(-\Lambda_{1}^{-1} F_{1}\right)^{i}, \cdots,\right. \\
\cdots, \ln \left(p_{\beta}, \Lambda_{\beta}\right)-\sum_{i=1}^{m_{\beta}-1} \frac{1}{i}\left(-\Lambda_{\beta}^{-1} F_{\beta}\right)^{i} \mid \\
\mid \ln \left(p_{\beta+1}, \lambda_{\beta+1}\right) I_{\beta+1}-\sum_{i=1}^{m_{\beta+1}-1} \frac{\left(-E_{\beta+1}\right)^{i}}{i \lambda_{\beta+1}^{i}}, \cdots, \\
\left.\cdots, \ln \left(p_{\alpha}, \lambda_{\alpha}\right) I_{\alpha}-\sum_{i=1}^{m_{\alpha}-1} \frac{\left(-E_{\alpha}\right)^{i}}{i \lambda_{\alpha}^{i}}\right] T
\end{array}
$$

where

$$
\begin{array}{r}
\ln \left(p_{i}, \Lambda_{i}\right)=: \operatorname{diag}\left[\begin{array}{cc}
\ln \left(r_{i}^{2}+g_{i}^{2}\right)+j 2 \pi p_{i} & -\tan ^{-1}\left(g_{i} / r_{i}\right) \\
\tan ^{-1}\left(g_{i} / r_{i}\right) & \ln \left(r_{i}^{2}+g_{i}^{2}\right)+j 2 \pi p_{i}
\end{array} \mid \ldots\right. \\
\left.\ldots \mid \begin{array}{cc}
\ln \left(r_{i}^{2}+g_{i}^{2}\right)+j 2 \pi p_{i} & -\tan ^{-1}\left(g_{i} / r_{i}\right) \\
\tan ^{-1}\left(g_{i} / r_{i}\right) & \ln \left(r_{i}^{2}+g_{i}^{2}\right)+j 2 \pi p_{i}
\end{array}\right]
\end{array}
$$

The proof for Lemma 3 is given in Appendix B to keep our arguments clean.

Lemma 3 Let $C \in \mathcal{F}^{n \times n}$ be nonsingular. The Jordan canonical form formula (5) satisfies $C=$ $e^{B}$. Furthermore, if $C$ is real, then $B$ in (6) satisfies $\bar{B} B=B \bar{B}$ and $e^{B+\bar{B}}=C^{2}$.

Lemma 4 Assume that $C \in \mathcal{R}^{n \times n}$ is nonsingular without negative eigenvalues. Let all logarithms of (6) mean their principal branches; that is, $p_{1}=\cdots=p_{\alpha}=0$. Then the matrix $B$ determined in the Jordan canonical form formula (6) is real and satisfies $C=e^{B}$.

Proof of Lemma 4 Since $C$ is real, there is a real nonsingular matrix $T$ transforming $C$ into a real Jordan canonical form. This, together with the principal branch assumption, all the terms in (6) are real since no eigenvalues of $C$ are negative. Then Lemma 3 yields the results.

\subsection{Matrix Logarithm by Hermite Interpolation Polynomials [8]}

Let $\lambda_{1}, \lambda_{2}, \cdots, \lambda_{\alpha}$ denote the distinctive nonzero eigenvalues of $C$ with multiplicities $m_{1}, m_{2}, \cdots$, $m_{\alpha}$ in its minimal polynomial $\Delta(C, s)$. Clearly, $m_{1}+\cdots+m_{\alpha}=\operatorname{deg}(\Delta(C, s))=: m$. How to fix the minimal polynomial $\Delta(C, s)$ is discussed in Theorem A1.4 [8, p. 504].

The so-called Hermite interpolation polynomial on the spectrum of $C$ is given by $H(s)=$ $h_{m-1} s^{m-1}+\cdots+h_{1} s+h_{0}$, where $h_{m-1}, \cdots, h_{0}$ are solutions to the following linear algebra equation system constructed with prescribed logarithm branches $p_{1}, \cdots, p_{\alpha}$.

$$
H^{(l)}\left(\lambda_{i}\right)=\ln ^{(l)}\left(p_{i}, \lambda_{i}\right), \quad l=0,1, \cdots, m_{i}-1, i=1, \cdots, \alpha, p_{i} \in \mathcal{Z}
$$

where $H^{(l)}\left(\lambda_{i}\right)$ means the $l$-th order derivative of $H(s)$ with respect to $s$ that is evaluated at $s=\lambda_{i}$, and thus $H^{(l)}\left(\lambda_{i}\right)$ is a polynomial in terms of $h_{m-1}, \cdots, h_{0}$ as appropriately. $\ln ^{(l)}\left(p_{i}, \lambda_{i}\right)$ can be interpreted similarly. Therefore, the above equations can be summarized in form of

$$
H\left(\lambda_{1}, m_{1} ; \cdots ; \lambda_{\alpha}, m_{\alpha}\right)\left[\begin{array}{c}
h_{m-1} \\
h_{m-2} \\
\vdots \\
\vdots \\
h_{1} \\
h_{0}
\end{array}\right]=\left[\begin{array}{c}
\frac{\vdots}{\ln ^{\left(m_{i}-1\right)}\left(p_{i}, \lambda_{i}\right)} \\
\vdots \\
\ln ^{(1)}\left(p_{i}, \lambda_{i}\right) \\
\frac{\ln ^{(0)}\left(p_{i}, \lambda_{i}\right)}{\vdots}
\end{array}\right]
$$


Clearly, $H\left(\lambda_{1}, m_{1} ; \cdots ; \lambda_{\alpha}, m_{\alpha}\right)$ is a non-singular matrix defined by the coefficients in the polynomials $H^{(l)}\left(\lambda_{i}\right), l=0,1, \cdots, m_{i}-1, i=1, \cdots, \alpha$. It has been claimed in [8] that $H(s)$ defined via (7) is unique whenever all the logarithm branches are specified.

Finally, we write the matrix logarithm algorithm corresponding to $C$ by

$$
B=: H(C)=h_{m-1} C^{m-1}+\cdots+h_{1} C+h_{0} I
$$

Lemma 5 If $C \in \mathcal{F}^{n \times n}$ is nonsingular, the Hermite interpolation polynomial (8) satisfies $C=e^{B}$. Furthermore, if $C$ is real, then it holds that $B \bar{B}=\bar{B} B$ and $e^{B+\bar{B}}=C^{2}$.

Proof of Lemma 5 See Theorem A1.9 and Corollary A1.11 [8, pp.509-511] for the details.

Lemma 6 Let $C \in \mathcal{R}^{n \times n}$ be nonsingular without negative eigenvalues. Let the logarithm branches of (7) be specified as in (9). Then, the matrix $B$ in (8) is real and satisfies $C=e^{B}$.

$$
\begin{cases}p_{i}=0, & \text { if } \lambda_{i} \text { is real } \\ p_{i}=\text { const } \in \mathcal{Z}, & \text { if } \lambda_{i} \text { is complex } \\ p_{i+1}=-p_{i}, & \text { if } \lambda_{i+1}=\overline{\lambda_{i}}\end{cases}
$$

Proof of Lemma 6 According to the logarithm branch specification in (9), the equations in (7) are nothing but equivalent re-writing of the following equations, each of which can be viewed as a polynomial equation in terms of $h_{m-1}, \cdots, h_{0}$ as appropriately.

$$
\begin{cases}H^{(l)}\left(\lambda_{i}\right)=\ln ^{(l)}\left(0, \lambda_{i}\right), & \text { if } \lambda_{i} \text { is real } \\ H^{(l)}\left(\lambda_{i}\right)=\ln ^{(l)}\left(p_{i}, \lambda_{i}\right), & \text { if } \lambda_{i} \text { is complex } \\ H^{(l)}\left(\lambda_{i+1}\right)=\ln ^{(l)}\left(-p_{i}, \lambda_{i+1}\right), & \text { if } \lambda_{i+1}=\overline{\lambda_{i}}\end{cases}
$$

where $l=0,1, \cdots, m_{i}-1$ and $i=1, \cdots, \alpha$. Obviously, if $\lambda_{i}$ is real, the corresponding polynomial equation is real. For a pair of conjugate eigenvalues, say $\lambda_{i}$ and $\lambda_{i+1}$, we obtain $H^{(l)}\left(\lambda_{i}\right)=$ $\ln ^{(l)}\left(p_{i}, \lambda_{i}\right)$ and $H^{(l)}\left(\lambda_{i+1}\right)=\ln ^{(l)}\left(-p_{i}, \lambda_{i+1}\right)\left(=\overline{\ln ^{(l)}\left(p_{i}, \lambda_{i}\right)}\right)$. This means that simple algebraic operations on $H^{(l)}\left(\lambda_{i}\right)=\ln ^{(l)}\left(p_{i}, \lambda_{i}\right)$ and $H^{(l)}\left(\lambda_{i+1}\right)=\ln ^{(l)}\left(-p_{i}, \lambda_{i+1}\right)$ can produce us two real polynomial equations. We can repeat the arguments for any conjugate eigenvalue pairs.

These arguments say that the above complex polynomial equations can always be equivalently changed into a group of real polynomial equations in terms of $h_{m-1}, \cdots, h_{0}$, which possess unique and real solutions $h_{m-1}, \cdots, h_{1}$ and $h_{0}$ (since (7) possesses unique solutions). Using this fact in (8) and noting that $C$ itself is real, the desired results follow.

\subsection{Remarks about Matrix Logarithm Lemmas}

Remark 1 No matter which algorithm is taken to get a matrix logarithm $B$ from $C$, namely, $C=e^{B}$, it is straightforward to see in all the lemmas that $B C=C B$ is true.

Remark 2 Logarithm branch problems appear in (3), (5), (6) and (7). This implies:

(i). $\ln (p, z)$ in (3) must be analytic over $\Omega$ for the Cauchy integration formula to make sense, and a specific logarithm branch should be prescribed. In other words, Eq. (3) reflects all the eigenvalues of $C$ on a single ramification of the Riemann surface;

(ii). In contrast, (5), (6) and (7) involve multiple logarithms that can be treated separately. In other words, (5), (6) and (7) may reflect the eigenvalues of $C$ on multiple ramifications on the Riemann surface if different branches are chosen for each individual logarithm. 
Remark 3 With a specific logarithm branch, $\ln (p, z)$ in (3) is analytic over $\Omega$ since $0 \notin \Omega$, and the Cauchy integral theorem [29] says that the integral (3) is independent of $\partial \Omega$. Thus the matrix $B$ of (3) is unique. With specific logarithm branches, uniqueness of the Hermite interpolation polynomial implies that the matrix $B$ of (7) is unique. However, even with fixed logarithm branches the matrix $B$ of (5) or (6) is generally not unique since the similarity transformation is not unique.

Remark 4 Lemmas 1, 3 and 5 are stated for both real and complex $C \in \mathcal{F}^{n \times n}$, while Lemmas 2, 4 and 6 are claimed only for real $C \in \mathcal{R}^{n \times n}$. To interpret them properly, we stress that:

(i). In Lemma 3, the matrix logarithm formula if $C$ is expressed via a complex Jordan canonical form is different from that if $C$ is real and expressed via a real Jordan canonical form;

(ii). In Lemmas 2 and 4, a principal logarithm branch specification is necessary in order to obtain a real matrix $B$. There is no such a principal logarithm branch constraint in Lemma 6 . This relaxation on logarithm branches is due to the proof approach [8, pp.507-510].

\section{Existence and Classification of Floquet Factorizations}

Consider the FDLCP system given by the homogeneous differential equation in $J \times \mathcal{F}^{n}$

$$
\dot{x}=A(t) x
$$

where $A(t)$ is $n \times n$, $h$-periodically time-varying; i.e., $A(t+h)=A(t) \in \mathcal{F}^{n \times n}$ for all $t \in J$.

Assume that $A(t)$ is locally integrable on $J$ if each of its elements is measurable in any Borel subsets of $J$ and $\|A(t)\|$ is Lebesgue integrable on each compact subinterval $\Gamma \subset J$, i.e., $\int_{\Gamma}\|A(t)\| d t<\infty$. This assumption is satisfied if $A(t)$ is continuous or piecewise continuous. This assumption allows us to deal with FDLCP systems with switching components.

\subsection{Definitions about Floquet Factorizations}

Definition 1 Let $\Phi(t, 0)$ denote the state transition matrix of the FDLCP system (10). If for some integer $k>0$, there exist

(i). a constant matrix $Q \in \mathcal{F}^{n \times n}$ with $\Phi(k h, 0)=e^{Q k h}$;

(ii). $P(t, 0) \in \mathcal{F}^{n \times n}$ with $P(0,0)=I$ and $P(t+k h, 0)=P(t, 0)$ for all $t$;

such that $\Phi(t, 0)=P(t, 0) e^{Q t}$ for all $t \in J$, then $P(t, 0) e^{Q t}$ is called a kh-periodic Floquet factorization of $\Phi(t, 0)$ with the Floquet factors $P(t, 0)$ and $Q$. If $P(t, 0)$ and $Q$ are real, $\Phi(t, 0)=P(t, 0) e^{Q t}$ is called a real Floquet factorization.

In the paper, $\Phi(k h, 0)$ is called the k-monodromy, while the 1-monodromy is conventionally termed the monodromy matrix of $A(t)$ [19] or the principal matrix of $A(t)$ [8, p.53].

Definition 2 In the FDLCP system (10), let $\Phi(t, 0)=P(t, 0) e^{Q t}$ be a kh-periodic Floquet factorization. Introduce the $k h$-fundamental region $\mathcal{C}_{f / k}$ on the complex plane as follows [33].

$$
\mathcal{C}_{f / k}:=\left\{z \in \mathcal{C}:-\omega_{h} /(2 k)<\operatorname{Im}(z) \leq \omega_{h} /(2 k)\right\}, \quad \omega_{h}=2 \pi / h
$$

If $\lambda(Q) \subset \mathcal{C}_{f / k}, \Phi(t, 0)=P(t, 0) e^{Q t}$ is termed a kh-periodic Floquet simplex.

Remark 5 In Definition $1, \Phi(k h, 0)=e^{Q k h}$ and $P(0,0)=I$ are required. There are FDLCP systems whose discompositions in form of $\Phi(t, 0)=P(t, 0) e^{Q t}$ exist but these conditions are not 
satisfied [19]. Without these conditions, eigenvalues of $Q$ may not be simply connected to those of the monodromy as we will see in Remark 7. This is somehow confusing.

If $Q$ is determined via the Cauchy integration formula, $\Phi(t, 0)=P(t, 0) e^{Q t}$ is termed a $\mathcal{C}$ Floquet factorization; If $Q$ is determined through a Jordan canonical form, $\Phi(t, 0)=P(t, 0) e^{Q t}$ is called a $\mathcal{J}$-Floquet factorization; If $Q$ is determined through a Hermite interpolation polynomial, $\Phi(t, 0)=P(t, 0) e^{Q t}$ is called an $\mathcal{H}$-Floquet factorization.

\subsection{Existence of $k h$-Periodic Floquet Factorizations}

The Floquet theorem and its modifications frequently encountered in the literature $[4,8,12,13$, 19, 20] can be viewed as a special case of Theorem 1 when $k=1$. Theorem 1 claims existence of $k h$-periodic Floquet factorizations in FDLCP systems.

Theorem 1 In the FDLCP system (10), assume that $A(t) \in \mathcal{F}^{n \times n}$ is locally integrable on $J$. For any integer $k>0, \Phi(t, 0)$ possesses a kh-periodic $\mathcal{C}$ - (resp., $\mathcal{J}$ - and $\mathcal{H}$-) Floquet factorization $\Phi(t, 0)=P(t, 0) e^{Q t}$, where $P(t, 0)$ is absolutely continuous, nonsingular for each $t \in J$, and $Q$ is possibly complex. More precisely, $Q$ is determined as follows.

(i) In Lemma 1, specify the logarithm branch $p$ and an open set $\Omega \subset \mathcal{C} \backslash\{0\}$ containing all eigenvalues of $\Phi^{k}(h, 0)$. Calculate $B$ by the Cauchy integration fromula (3) with $C=\Phi^{k}(h, 0)$.

(ii) In Lemma 3, specify the logarithm branches $p_{1}, \cdots, p_{\alpha}$. Let the matrix $B$ be given by the Jordan canonical formula (5) with $C=\Phi^{k}(h, 0)$.

(iii) In Lemma 5, specify the logarithm branches $p_{1}, \cdots, p_{\alpha}$ and determine the Hermite interpolation polynomial $H(s)$ by the eigenvalues of $\Phi^{k}(h, 0)$ as in (7). Let the matrix $B$ be given by the Hermite interpolation polynomial (8) evaluated with $C=\Phi^{k}(h, 0)$.

Then a kh-periodic $\mathcal{C}$ - (resp., $\mathcal{J}$ - and $\mathcal{H}$-) Floquet factorization is given by $Q=(k h)^{-1} B$ and $P(t, 0)=\Phi(t, 0) e^{-Q t}$. Moreover, the system is asymptotically stable if and only if all eigenvalues of $Q$ have negative real parts, that is, $\operatorname{Re} \lambda(Q)<0$; or equivalently, the absolute values of all the eigenvalues of $\Phi(h, 0)$ are strictly less than 1 , that is, $\mid \lambda(\Phi(h, 0) \mid<1$.

Remark 6 By Remarks 2 and 3, kh-periodic $\mathcal{C}$-, $\mathcal{J}$-and $\mathcal{H}$-Floquet factorizations are not unique due to logarithm branch problems. As for the Cauchy integration formula (3), there are many ways to define integral paths. The Cauchy integral theorem [26] says that $Q$ is independent of the integral path $\partial \Omega$ as long as $\Omega$ is defined appropriately.

Remark 7 The eigenvalues of $\Phi(h, 0)$, say $\sigma_{1}, \sigma_{2}, \cdots, \sigma_{n}$, are called characteristic multipliers. Then the eigenvalues of $\Phi^{k}(h, 0)$ are $\sigma_{1}^{k}, \sigma_{2}^{k}, \cdots, \sigma_{n}^{k}$. In a kh-periodic Floquet factorization $\Phi(t, 0)=P(t, 0) e^{Q t}$, denote the eigenvalues of $Q$ by $\lambda_{1}, \lambda_{2}, \cdots, \lambda_{n}$, which are called $k$ characteristic exponents. If properly enumerated, $\sigma_{1}^{k}=e^{\lambda_{1} k h}, \sigma_{2}^{k}=e^{\lambda_{2} k h}, \cdots, \sigma_{n}^{k}=e^{\lambda_{n} k h}$. Note that $e^{\lambda_{i} k h}=e^{\lambda_{i} k h+j 2 p_{i} \pi}=e^{\left(\lambda_{i}+j 2 p_{i} \pi /(k h)\right) k h}$ for any $m \in \mathcal{Z}$ and $i=1,2, \cdots, n$. Thus, $\lambda_{1}, \lambda_{2}, \cdots$, $\lambda_{n}$ are not uniquely determined by $\sigma_{1}^{k}, \sigma_{2}^{k}, \cdots, \sigma_{n}^{k}$ (only determined $\bmod (j 2 \pi /(k h))$ ). We have

$$
\operatorname{Re}\left(\lambda_{i}\right)=(k h)^{-1} \operatorname{Re}\left(\ln \left(p_{i}, \sigma_{i}^{k}\right)\right)=(k h)^{-1} \operatorname{Re}\left(\ln \left(\sigma_{i}^{k}\right)\right), \quad i=1,2, \cdots, n
$$

which are uniquely determined by $A(t)$. By Theorem 6.3.2 [19], $\Phi(t, 0)$ is unique for each $t$ under the assumption on $A(t)$. Thus, $\Phi(h, 0)$ is uniquely determined and so are its eigenvalues. 
Eq. (11) is meant in the eigenvalue magnitude. If multiplicities of $\lambda_{i}$ and $\sigma_{i}^{k}$ are concerned, Theorem A1.12 [8] says that multiplicity of $\sigma_{i}^{k}$ in the minimal polynomial of $\Phi^{k}(h, 0)$ is equal to that of $\lambda_{i}$ in the minimal polynomial of $Q$. Also, the eigenvector of $\Phi^{k}(h, 0)$ corresponding to $\sigma_{i}^{k}$ is an eigenvector of $Q$ corresponding to $\lambda_{i}$. Corollary A1.8 [8] coincides with this observation.

Remark 8 If the monodromy eigenvalues, $\sigma_{1}, \sigma_{2}, \cdots, \sigma_{n}$, satisfy $\left|\sigma_{1}\right|<1,\left|\sigma_{2}\right|<1, \cdots,\left|\sigma_{n}\right|<1$, then $\left|\sigma_{1}^{k}\right|<1,\left|\sigma_{2}^{k}\right|<1, \cdots,\left|\sigma_{n}^{k}\right|<1$ for any $k \geq 1$. Hence, asymptotic stability of the FDLCP system (10) will be reflected in any kh-periodic Floquet factorizations. Note that for any $t, \tau \in J$, $\Phi(t, \tau)=\Phi(t, 0) \Phi^{-1}(\tau, 0)$. It follows that $\Phi(t, \tau)=P(t, 0) e^{Q(t-\tau)} P^{-1}(\tau, 0)$. Letting $P(t)=P(t, 0)$, we have $\Phi(t, \tau)=P(t) e^{Q(t-\tau)} P^{-1}(\tau)$, which is also frequently used in the literature.

\subsection{Existence of $2 k h$-Periodic Real Floquet Factorizations}

In Theorem 1, Floquet factorizations are usually expressed with complex Floquet factors, even if $A(t)$ itself is real. This may bring us difficulties in applications. In this section, we see that real Floquet factorizations are also available in real FDLCP systems but in the $2 k h$-periodic sense.

Theorem 2 In the FDLCP system (10), assume that $A(t) \in \mathcal{R}^{n \times n}$ is locally integrable on J. For any integer $k>0, \Phi(t, 0)$ has a $2 k h$-periodic real $\mathcal{C}$ - (resp., $\mathcal{J}$ - and $\mathcal{H}$-) Floquet factorization $\Phi(t, 0)=P(t, 0) e^{Q t}$, where $P(t, 0)$ is absolutely continuous and nonsingular for each $t \in J$. More precisely, $Q$ can be determined as follows.

(i) Assume that $\Omega$ and $\partial \Omega$ are defined as in Theorem 1. In Lemma 1, specify the logarithm branch $p$ and evaluate (3) with $C=\Phi^{k}(h, 0)$.

(ii) In Lemma 3, specify the logarithm branches $p_{1}, \cdots, p_{\alpha}$. Evaluate (6) with $C=\Phi^{k}(h, 0)$.

(iii) In Lemma 5, specify the logarithm branches $p_{1}, \cdots, p_{\alpha}$ and fix the Hermite interpolation polynomial $H(s)$ by the eigenvalues of $\Phi^{k}(h, 0)$ via (7). Evaluate (8) with $C=\Phi^{k}(h, 0)$.

Then a $2 k h$-periodic real $\mathcal{C}$ - (resp., $\mathcal{J}$ - and $\mathcal{H}$-) Floquet factorization is given by the Floquet factors $Q=(2 k h)^{-1}(B+\bar{B})$ and $P(t, 0)=\Phi(t, 0) e^{-Q t}$.

Proof of Theorem 2 Only the $\mathcal{C}$-Floquet factorization case is proved. Lemma 1 leads that there is a (possibly complex) matrix $B$ given by (3) such that $\Phi(k h, 0)=e^{2 k h B}$ and $B \bar{B}=\bar{B} B$. This, together with $(29)$ and that $\Phi(k h, 0)$ is real, yields

$$
\begin{aligned}
e^{2 k h B} e^{2 k h \bar{B}} & =e^{2 k h(B+\bar{B})}=\Phi(k h, 0) \bar{\Phi}(k h, 0)=\Phi(k h, 0) \Phi(k h, 0) \\
& =\Phi(2 k h, k h) \Phi(k h, 0)=\Phi(2 k h, 0)
\end{aligned}
$$

where we used $\Phi(k h, 0)=\Phi(2 k h, k h)$, which again follows from the $h$-periodicity of $A(t)$. Eq. (12) implies that $e^{2 k h Q}=\Phi(2 k h, 0)$. Now we view $A(t)$ to be $2 k h$-periodic. Then by means of $e^{2 k h Q}=$ $\Phi(2 k h, 0)$ and repeating the proof of Theorem 1 we see that $\Phi(t, 0)=P(t, 0) e^{Q t}$, where $P(t, 0):=$ $\Phi(t, 0) e^{-Q t}$ is real, $2 k h$-periodic, and $Q=(2 k h)^{-1}(B+\bar{B})$ is real.

Remark 9 To work out a real 2kh-periodic Floquet factorization by Theorem 2, one must view the original system to be $2 k h$-periodic. This turns out to be an obstacle in some applications [20]. To surmount this, there are efforts to directly determine h-periodic real Floquet factorizations for real FDLCP systems. Typical results are reported in [5],[20]. In the paper, we try to establish $k h$-periodic real Floquet factorizations by exploiting the results of Lemmas 2, 4 and 6 . 


\subsection{Existence of $k h$-Periodic Real Floquet Factorizations}

Now we show that $k h$-periodic real Floquet factorizations are also possible if eigenvalue conditions on the $k$-monodromy are strengthened slightly, beside $A(t) \in \mathcal{R}^{n \times n}$. Theorem 3 can be shown by repeating arguments about Theorem 1 but based on Lemmas 2, 4 and 6 .

Theorem 3 In the FDLCP system (10), assume that $A(t) \in \mathcal{R}^{n \times n}$ is locally integrable on $J$. For any integer $k>0, \Phi(t, 0)$ has a kh-periodic real $\mathcal{C}$ - (resp., $\mathcal{J}$-and $\mathcal{H}$-) Floquet factorization $\Phi(t, 0)=P(t, 0) e^{Q t}$, where $P(t, 0)$ is absolutely continuous and nonsingular for each $t \in J$, if $\Phi^{k}(h, 0)$ has no negative eigenvalues, and $Q$ is determined accordingly.

(i) Assume that $\Omega$ and $\partial \Omega$ are defined in Theorem 1. In Lemma 2, take the principal logarithm branch $(p=0)$ and evaluate the Cauchy integration formula (3) with $C=\Phi^{k}(h, 0)$.

(ii) In Lemma 4, take the principal logarithm branches $\left(p_{1}=\cdots=p_{\alpha}=0\right)$. Let the Jordan canonical form formula (6) be evaluasted with $C=\Phi^{k}(h, 0)$.

(iii) In Lemma 6, specify the logarithm branches $p_{1}, \cdots, p_{\alpha}$ as described in (9) and determine the Hermite interpolation polynomial $H(s)$ by the eigenvalues of $\Phi^{k}(h, 0)$ through (7). Evaluate the Hermite interpolation polynomial (8) with $C=\Phi^{k}(h, 0)$.

Then a kh-periodic $\mathcal{C}$ - (resp., $\mathcal{J}$ - and $\mathcal{H}$-) real Floquet factorization is given by the Floquet factors $Q=(k h)^{-1} B$ and $P(t, 0)=\Phi(t, 0) e^{-Q t}$.

\subsection{Existence of $k h$-Periodic Floquet Simplices}

Theorem 4 In the FDLCP system (10), assume that $A(t) \in \mathcal{F}^{n \times n}$ is locally integrable on $J$. Then there always exists a kh-periodic Floquet simplex $\Phi(t, 0)=P(t, 0) e^{Q t}$, where $P(t, 0)$ is absolutely continuous and nonsingular for each $t \in J$, and $\lambda(Q) \subset \mathcal{C}_{f / k}$.

Proof of Theorem 4 By Theorem 1 , there is a $k h$-periodic Floquet factorization $\Phi(t, 0)=$ $\tilde{P}(t, 0) e^{\tilde{Q} t}$, with $e^{\tilde{Q} k h}=\Phi(k h, 0)$. Express $\tilde{Q}$ via a Jordan canonical form $\tilde{Q}=S \tilde{J} S^{-1}$. Here $S$ is nonsingular, and $\tilde{J}=\operatorname{diag}\left[\tilde{J}_{1}, \tilde{J}_{2}, \cdots, \tilde{J}_{\alpha}\right]$ with $\tilde{J}_{i}$ being a $n_{i} \times n_{i}$ Jordan block corresponding to an eigenvalue $\tilde{\lambda}_{i}$ of $\tilde{Q}$. Clearly, $e^{\tilde{Q} t}=S e^{\tilde{J} t} S^{-1}$, where $e^{\tilde{J} t}=\operatorname{diag}\left[e^{\tilde{J}_{1} t}, e^{\tilde{J}_{2} t}, \cdots, e^{\tilde{J}_{\alpha} t}\right]$ and

$$
e^{\tilde{J}_{i} t}=e^{\tilde{\lambda}_{i} t}\left[\begin{array}{ccccc}
1 & t & \frac{t}{2} & \cdots & \frac{t^{n_{i}-1}}{\left(n_{i}-1\right) !} \\
0 & 1 & t & \cdots & \frac{t^{n_{i}-2}}{\left(n_{i}-2\right) !} \\
\vdots & \vdots & \vdots & \ddots & \vdots \\
0 & 0 & 0 & \cdots & 1
\end{array}\right]
$$

Write $\tilde{\lambda}_{i}=\lambda_{i}+j k_{i} \omega_{k h}, k_{i} \in \mathcal{Z}, i=1,2, \cdots, \alpha$ with $\lambda_{i} \in \mathcal{C}_{f / k}$. This is always possible. By (13), $e^{\tilde{J}_{i} k h}=e^{J_{i} k h}$ for each $i$ where $J_{i}$ is a Jordan block in term of $\lambda_{i}$. We have that $e^{\tilde{Q} k h}=$ $S e^{J k h} S^{-1}=e^{S J S^{-1} k h}=e^{Q k h}$, in which $Q:=S J S^{-1}$ and $J:=\operatorname{diag}\left[J_{1}, J_{2}, \cdots, J_{\alpha}\right]$. Clearly, $Q$ has eigenvalues $\lambda_{1}, \lambda_{2}, \cdots, \lambda_{\alpha}$ in $\mathcal{C}_{f / k}$. Now we repeat the proof arguments of Theorem 1 in term of $\Phi(k h, 0)=e^{Q k h}$. Then the desired assertion follows.

Remark 10 In the proof, $\tilde{Q}$ can be determined from any kh-periodic Floquet factorizations. Floquet simplices are needed in harmonic controllability criteria in the FDLCP setting [34]. 


\subsection{Existence of $k h$ - and $2 k h$-Periodic Real Floquet Simplices}

Theorem 5 In the FDLCP system (10), let $A(t) \in \mathcal{R}^{n \times n}$ be locally integrable on $J$. For any integer $k>0$ we always have a $2 k$-periodic real Floquet simplex $\Phi(t, 0)=P(t, 0) e^{Q t}$, where $P(t, 0)$ is absolutely continuous and nonsingular for each $t \in J$, and $\lambda(Q) \subset \mathcal{C}_{f / 2 k}$.

Proof of Theorem 5 By Theorem $2, \Phi(t, 0)$ has a $2 k h$-periodic real Floquet simplex $\Phi(t, 0)=$ $\tilde{P}(t, 0) e^{\tilde{Q} t}$. Let $\tilde{Q}=S \tilde{J} S^{-1}$ be a real Jordan canonical form with $S$ nonsingular and real, and $\tilde{J}=\operatorname{diag}\left[\tilde{R}_{1}, \cdots, \tilde{R}_{\beta}, \tilde{J}_{\beta+1}, \cdots, \tilde{J}_{\alpha}\right] . \quad \tilde{R}_{i}$ is a real Jordan block for a pair of complex conjugate eigenvalues $\tilde{r}_{i} \pm j \tilde{g}_{i}$, and $\tilde{J}_{i}$ is a real Jordan block corresponding to a real eigenvalue. Exact definitions for $\tilde{R}$ and $\tilde{J}_{i}$ can be found in Section 2.2 .

The proof is similar to that of Theorem 4 by using $\tilde{R}_{i}$ and $\tilde{J}_{i}$. Our attention is focused only on $\tilde{R}_{i}=\tilde{\Lambda}_{i}+\tilde{F}_{i}$. It is straightforward to see by direct computations that

$$
e^{\tilde{R}_{i} t}=e^{\tilde{\Lambda}_{i} t}\left[\sum_{l=1}^{\tilde{m}_{i}-1} \frac{1}{l !} t^{l} \tilde{F}_{i}^{l}\right]
$$

where $\tilde{m}_{i}$ is the least non-negative integer such that $\tilde{F}_{i}^{l}=0$ for all $l \geq \tilde{m}_{i}$. Also, we have

$$
e^{\tilde{\Lambda}_{i} t}=\operatorname{diag}\left[\begin{array}{cc|c|cc}
e^{\tilde{r}_{i} t} \cos \left(\tilde{g}_{i} t\right) & e^{\tilde{r}_{i} t} \sin \left(\tilde{g}_{i} t\right) & \ldots & e^{\tilde{r}_{i} t} \cos \left(\tilde{g}_{i} t\right) & e^{\tilde{r}_{i} t} \sin \left(\tilde{g}_{i} t\right) \\
-e^{\tilde{r}_{i} t} \sin \left(\tilde{g}_{i} t\right) & e^{\tilde{r}_{i} t} \cos \left(\tilde{g}_{i} t\right) & & -e^{\tilde{r}_{i} t} \sin \left(\tilde{g}_{i} t\right) & e^{\tilde{r}_{i} t} \cos \left(\tilde{g}_{i} t\right)
\end{array}\right]
$$

Now write $\tilde{r}_{i}+j \tilde{g}_{i}=\tilde{r}_{i}+j\left(\tilde{g}_{0 i}+k_{i} \omega_{2 k h}\right), k_{i} \in \mathcal{Z}$ with $\tilde{r}_{i}+j \tilde{g}_{0 i} \in \mathcal{C}_{f / 2 k}$. Then, (14) leads that $e^{\tilde{R}_{i} 2 k h}=e^{R_{i} 2 k h}$ for each $i$ where $R_{i}$ is a real Jordan block in term of $\tilde{r}_{i}+j \tilde{g}_{0 i}$. Furthermore, we have that $e^{\tilde{Q} 2 k h}=S e^{J 2 k h} S^{-1}=e^{S J S^{-1} 2 k h}=e^{Q 2 k h}$, in which $Q:=S J S^{-1}$ and $J:=\operatorname{diag}\left[R_{1}, \cdots, R_{\beta}, J_{\beta+1}, \cdots, J_{\alpha}\right]$ is real. All the eigenvalues of $Q$ are in $\mathcal{C}_{f / 2 k}$.

Finally, repeating the proof of Theorem 1 in term of $\Phi(2 k h, 0)=e^{Q 2 k h}$ yields the assertion.

Based on Theorems 3, 4 and 5, Theorem 6 can be shown.

Theorem 6 In the FDLCP system (10), assume that $A(t) \in \mathcal{R}^{n \times n}$ is locally integrable on $J$. For an integer $k>0, \Phi^{k}(h, 0)$ satisfies the eigenvalue conditions in Theorem 3 as appropriately. Then, a kh-periodic real Floquet factorization $\Phi(t, 0)=\tilde{P}(t, 0) e^{\tilde{Q} t}$ exists, from which a kh-periodic real Floquet simplex $\Phi(t, 0)=P(t, 0) e^{Q t}$ can be derived, where $P(t, 0)$ is absolutely continuous and nonsingular for each $t \in J$, and $\lambda(Q) \subset \mathcal{C}_{f / k}$.

\section{Characteristics of Floquet Factors $P(t, 0)$ and $Q$}

\subsection{Harmonic Properties about $P(t, 0)$}

Let $\Phi(t, 0)=P(t, 0) e^{Q t}$ be a $k h$-periodic Floquet factorization. Theorem 6.3 .2 [19] yields

$$
\left\{\begin{array}{l}
P(t, 0)=\Phi(t, 0) e^{-Q t}, \quad \dot{P}(t, 0)=[A(t) \Phi(t, 0)-\Phi(t, 0) Q] e^{-Q t} \\
P^{-1}(t, 0)=e^{Q t} \Phi(0, t), \quad \dot{P}^{-1}(t, 0)=e^{Q t}[Q \Phi(0, t)-\Phi(0, t) A(t)]
\end{array}\right.
$$

for a.e. $t \in[0, k h)$. In the paper, we denote $\dot{P}^{-1}(t, 0)=: d\left(P^{-1}(t, 0)\right) / d t$ and $\ddot{P}^{-1}(t, 0)=$ : $d^{2}\left(P^{-1}(t, 0)\right) / d t^{2}$. By $(15)$ and Fourier theory [3, 30], we claim some harmonic features of $P(t, 0)$.

Theorem 7 In the FDLCP system (10), we have 
(i) If $A(t)$ is differentiable a.e. in $[0, h)$, then $P(t, 0)$ and $P^{-1}(t, 0)$ are absolutely continuous, while $\dot{P}(t, 0)$ and $\dot{P}^{-1}(t, 0)$ are differentiable a.e. in $[0, k h)$. Hence, the Fourier series of $P(t, 0)$ and $P^{-1}(t, 0)$ converge a.e. in $[0, k h)$;

(ii) If $A(t)$ is piecewise continuous and differentiable a.e. in $[0, h)$, then $P(t, 0)$ and $P^{-1}(t, 0)$ are absolutely continuous, and $\dot{P}(t, 0)$ and $\dot{P}^{-1}(t, 0)$ are piecewise continuous. Therefore, the Fourier series of $P(t, 0)$ and $P^{-1}(t, 0)$ are absolutely and uniformly convergent in $[0, h k)$, while the Fourier series of $\dot{P}(t, 0)$ and $\dot{P}^{-1}(t, 0)$ converge a.e. in $[0, k h)$;

(iii) If $A(t)$ is continuous and $d A(t) / d t$ is piecewise continuous in $[0, h)$, then $P(t, 0)$ and $P^{-1}(t, 0)$ are absolutely continuous, $\dot{P}(t, 0)$ and $\dot{P}^{-1}(t, 0)$ are continuous while $\ddot{P}(t, 0)$ and $\ddot{P}^{-1}(t, 0)$ are piecewise continuous. Also, the Fourier series of $P(t, 0), P^{-1}(t, 0), \dot{P}(t, 0)$ and $\dot{P}^{-1}(t, 0)$ are absolutely and uniformly convergent in $[0, k h)$, while those of $\ddot{P}(t, 0)$ and $\ddot{P}^{-1}(t, 0)$ converge a.e. in $[0, k h)$; the Fourier series of $\dot{P}(t, 0)$ and $\dot{P}^{-1}(t, 0)$ are equal to termwise differentiation of those of $P(t, 0)$ and $P^{-1}(t, 0)$, respectively.

The next theorem collects facts about Floquet factorizations whose $P(t, 0)$ possesses only finitely many harmonic waves. A proof can be given based on the results in [34].

Theorem 8 In the FDLCP system (10), assume that $A(t) \in \mathcal{F}^{n \times n}$ is locally integrable on $J$. Suppose that a kh-periodic Floquet factorization $\Phi(t, 0)=\tilde{P}(t, 0) e^{\tilde{Q} t}$ satisfies

$$
\tilde{P}(t, 0)=\sum_{|i| \leq N_{p}} \tilde{P}_{i} e^{j i \omega_{k h} t}, \quad \lambda(\tilde{Q}) \subset \bigcup_{|i| \leq N_{q}}\left\{\mathcal{C}_{f / k}+j i \omega_{k h}\right\}, \quad \omega_{k h}=\omega_{h} / k
$$

for some integers $N_{p} \geq 0$ and $N_{q} \geq 0$; that is, the Fourier series of $\tilde{P}(t, 0)$ contains finitely many harmonic waves, and all eigenvalues of $\tilde{Q}$ belong to a horizontally strip region along $\mathcal{C}_{f / k}$. Then, there exists a kh-periodic Floquet simplex $\Phi(t, 0)=P(t, 0) e^{Q t}$ such that

$$
P(t, 0)=\sum_{|i| \leq N_{p}+N_{q}} P_{i} e^{j i \omega_{k h} t}, \quad \lambda(Q) \subset \mathcal{C}_{f / k}
$$

Here $\tilde{P}_{k}$ and $P_{k}$ denote the Fourier coefficients of $\tilde{P}(t, 0)$ and $P(t, 0)$, respectively.

Remark 11 The properties of Theorem 7 help us in deriving essential features about frequency response operators of FDLCP systems [30],[32]. In addition, Theorem 8 can be proved independently of how the original kh-periodic Floquet factorization is determined.

\subsection{Properties about $Q$}

Now we consider relationships about the Floquet factor $Q$ among $\mathcal{C}$-, $\mathcal{J}$ - and $\mathcal{H}$-Floquet factorizations obtained via Theorem 1. A proof for Theorem 9 is given in Appendix C.

Theorem 9 In the FDLCP system (10), assume that $A(t) \in \mathcal{F}^{n \times n}$ is h-periodic, locally integrable on $J$. The following assertions hold for Floquet factorizations defined in Theorem 1.

(i) Let $\Phi(t, 0)=P_{1}(t, 0) e^{Q_{1} t}$ and $\Phi(t, 0)=P_{2}(t, 0) e^{Q_{2} t}$ be kh-periodic $\mathcal{C}$-Floquet factorizations given by the Cauchy integration formula (3) in the $p_{1}$-th and $p_{2}$-th logarithm branches, respectively. Then, $Q_{1}-Q_{2}=(k h)^{-1} j 2 \pi\left(p_{1}-p_{2}\right) I$ and $Q_{1} \neq Q_{2}$ as long as $p_{1} \neq p_{2}$; 
(ii) Let $\Phi(t, 0)=P_{1}(t, 0) e^{Q_{1} t}$ and $\Phi(t, 0)=P_{2}(t, 0) e^{Q_{2} t}$ be kh-periodic $\mathcal{J}$-Floquet factorizations given by (5) under a same Jordan canonical form $\Phi^{k}(h, 0)=T^{-1} J_{\Phi^{k}(h, 0)} T$ but the logarithms in (5) are specified, respectively, with $p_{1}, \cdots, p_{\alpha}$ and $p_{1}^{\prime}, \cdots, p_{\alpha}^{\prime}$. Then, $Q_{1}-Q_{2}=$ $(k h)^{-1} j 2 \pi T^{-1} \operatorname{diag}\left[\left(p_{1}-p_{1}^{\prime}\right)_{m_{1}}, \cdots,\left(p_{\alpha}-p_{\alpha}^{\prime}\right)_{m_{\alpha}}\right] T$, where $\left(p_{i}-p_{i}^{\prime}\right)_{m_{i}}=: \operatorname{diag}\left[p_{i}-p_{i}^{\prime}, \cdots, p_{i}-\right.$ $\left.p_{i}^{\prime}\right] \in \mathcal{R}^{m_{i} \times m_{i}}$. Moreover, $Q_{1} \neq Q_{2}$ as long as $\left(p_{1}, \cdots, p_{\alpha}\right) \neq\left(p_{1}^{\prime}, \cdots, p_{\alpha}^{\prime}\right)$;

(iii) Let $\Phi(t, 0)=P_{1}(t, 0) e^{Q_{1} t}$ and $\Phi(t, 0)=P_{2}(t, 0) e^{Q_{2} t}$ be kh-periodic $\mathcal{H}$-Floquet factorizations determined by (7) and (8) and the logarithms in (7) are specified with $p_{1}, \cdots, p_{\alpha}$ and $p_{1}^{\prime}$, $\cdots, p_{\alpha}^{\prime}$, respectively. Then, $Q_{1}-Q_{2}=(k h)^{-1} j 2 \pi \sum_{i=0}^{m-1} \xi_{i}\left(\Phi^{k}(h, 0)\right)^{i}$, where the coefficients $\xi_{m-1}, \cdots, \xi_{0}$ are given by

$$
\left.\left[\begin{array}{c}
\xi_{m-1} \\
\xi_{m-2} \\
\vdots \\
\vdots \\
\xi_{1} \\
\xi_{0}
\end{array}\right]=H^{-1}\left(\lambda_{1}, m_{1} ; \cdots ; \lambda_{\alpha}, m_{\alpha}\right)\left[\begin{array}{c}
\vdots \\
\hline 0 \\
\vdots \\
0 \\
\frac{p_{i}-p_{i}^{\prime}}{\vdots}
\end{array}\right]\right\} \quad m_{i} \text { rows, } i=1, \cdots, \alpha
$$

Also, $Q_{1} \neq Q_{2}$ as long as $\left(p_{1}, \cdots, p_{\alpha}\right) \neq\left(p_{1}^{\prime}, \cdots, p_{\alpha}^{\prime}\right)$.

Moreover, in each case it is satisfied that $P_{1}(t, 0)=P_{2}(t, 0) e^{\left(Q_{2}-Q_{1}\right) t}$.

Eigenvalue characteristics between the $k$-monodromy and $Q$ are argued in Remark 7 . The following theorem reveals other features, which is proved in Appendix D.

Theorem 10 In the FDLCP system (10), assume that $A(t) \in \mathcal{F}^{n \times n}$ is locally integrable and that $\Phi(t, 0)=P(t, 0) e^{Q t}$ is a kh-periodic Floquet factorization. Then for any integer $k \geq 1$, it holds

$$
[\operatorname{det}(\Phi(h, 0))]^{k}=\left(\prod_{i} \lambda_{i}(\Phi(h, 0))\right)^{k}=\exp \left\{k h \sum_{i} \lambda_{i}(Q)\right\}
$$

where $\lambda_{i}(\cdot)$ denotes the $i$-th eigenvalue of a matrix $(\cdot)$. Furthermore, we have

(i) If $\Phi(t, 0)=P(t, 0) e^{Q t}$ is a kh-periodic $\mathcal{C}$-Floquet factorization obtained in Theorem 1, then $\lambda_{i}(Q)=(k h)^{-1} \ln \left(p, \lambda_{i}^{k}(\Phi(h, 0))\right)$;

(ii) If $\Phi(t, 0)=P(t, 0) e^{Q t}$ is a kh-periodic $\mathcal{J}$-Floquet factorization in Theorem 1 , then $\lambda_{i}(Q)=$ $(k h)^{-1} \ln \left(p_{i}, \lambda_{i}^{k}(\Phi(h, 0))\right)$ where $p_{i}$ is meant according to the corresponding Jordan block in the Jordan canonical form of $\Phi^{k}(h, 0)$ (instead of the Jordan canonical form of $\Phi(h, 0)$ ).

(iii) If $\Phi(t, 0)=P(t, 0) e^{Q t}$ is a kh-periodic $\mathcal{H}$-Floquet factorization given by Theorem 1 , then $\lambda_{i}(Q)=(k h)^{-1} H\left(\lambda_{i}^{k}(\Phi(h, 0))\right)$.

\subsection{Properties between $P(t, 0)$ and $Q$}

Now we examine features involving both $P(t, 0)$ and $Q$ to get a more complete picture. 
Theorem 11 In the FDLCP system (10), assume that $A(t) \in \mathcal{F}^{n \times n}$ is h-periodic, locally integrable in $J$. Let $\Phi(t, 0)=P(t, 0) e^{Q t}$ be a kh-periodic Floquet factorization. Then

$$
\left\{\begin{array}{l}
\sum_{i} \lambda_{i}(Q)=\frac{1}{h} \int_{0}^{h} \operatorname{tr}(A(\tau)) d \tau-\frac{1}{k h} \int_{o}^{k h} \operatorname{tr}\left(P^{-1}(\tau, 0) \dot{P}(\tau, 0)\right) d \tau \\
{\left[\int_{0}^{k h} P(\tau, 0) d \tau\right] Q=\int_{0}^{k h} A(\tau) P(\tau, 0) d \tau}
\end{array}\right.
$$

and

$$
\left\{\begin{array}{l}
\sum_{i} \lambda_{i}(Q)=\frac{1}{h} \int_{0}^{h} \operatorname{tr}(A(\tau)) d \tau+\frac{1}{k h} \int_{o}^{k h} \operatorname{tr}\left(P(\tau, 0) \dot{P}^{-1}(\tau, 0)\right) d \tau \\
Q\left[\int_{0}^{k h} P^{-1}(\tau, 0) d \tau\right]=\int_{0}^{k h} P^{-1}(\tau, 0) A(\tau) d \tau
\end{array}\right.
$$

where $\dot{P}^{-1}(t, 0)=: d\left(P^{-1}(t, 0)\right) / d t$.

Proof of Theorem 11 By means of (15), it follows readily that

$$
P(t, 0) Q=A(t) P(t, 0)-\dot{P}(t, 0), \quad \text { a.e., } t \in[0, k h)
$$

Or equivalently, we write

$$
Q=P^{-1}(t, 0) A(t) P(t, 0)-P^{-1}(t, 0) \dot{P}(t, 0), \quad \text { a.e., } t \in[0, k h)
$$

Based on (19) and (20), let us show the first equation of (17). We observe

$$
\begin{aligned}
& \sum_{i} \lambda_{i}(Q)=\operatorname{tr}(Q) \\
= & \frac{1}{k h} \operatorname{tr}\left(\int_{0}^{k h} P^{-1}(\tau, 0) A(\tau) P(\tau, 0) d \tau\right)-\frac{1}{k h} \operatorname{tr}\left(\int_{0}^{k h} P^{-1}(\tau, 0) \dot{P}(\tau, 0) d \tau\right) \\
= & \frac{1}{k h} \int_{0}^{k h} \operatorname{tr}(A(\tau)) d \tau-\frac{1}{k h} \int_{0}^{k h} \operatorname{tr}\left(P^{-1}(\tau, 0) \dot{P}(\tau, 0)\right) d \tau
\end{aligned}
$$

The assertion follows since $A(t)$ is $h$-periodic and thus $\int_{0}^{k h} \operatorname{tr}(A(\tau)) d \tau=k \int_{0}^{h} \operatorname{tr}(A(\tau)) d \tau$.

Next, integrating both sides of (19), we are led that

$$
\begin{aligned}
& \int_{0}^{k h} A(\tau) P(\tau, 0) d \tau=\int_{0}^{k h} P(\tau, 0) Q d \tau+\int_{0}^{k h} \dot{P}(\tau, 0) d \tau \\
= & \int_{0}^{k h} P(\tau, 0) d \tau Q+\int_{0}^{k h} d P(\tau, 0)=\int_{0}^{k h} P(\tau, 0) d \tau Q
\end{aligned}
$$

since $P(k h, 0)=P(0,0)$. This yields the second equation of $(17)$.

To show (18), we mention the facts that $P(t, 0) Q P^{-1}(t, 0)=A(t)-\dot{P}(t, 0) P^{-1}(t, 0)$ and $\dot{P}(t, 0) P^{-1}(t, 0)+P(t, 0) \dot{P}^{-1}(t, 0)=0$. Thus it follows that $Q P^{-1}(t, 0)=P^{-1}(t, 0) A(t)+\dot{P}^{-1}(t, 0)$, based on which the assertion is derived by repeating similar arguments.

\section{Properties among Floquet Facotrizations}

\subsection{Further Definitions about Floquet Factorizations}

Definition 3 In the system (10), let $\Phi(t, 0)=P(t, 0) e^{Q t}$ be a kh-periodic Floquet factorization.

(i) If $P(t, 0) e^{Q t}=e^{Q t} P(t, 0)$, it is said that $\Phi(t, 0)=P(t, 0) e^{Q t}$ is commutative; 
(ii) If there are nonzero matrices $Q_{r}$ and $Q_{\bar{r}}$ such that

$$
\begin{cases}Q=Q_{\bar{r}}+Q_{r}, & e^{Q t}=e^{Q_{r} t} e^{Q_{\bar{r}} t}, \quad \forall t \in J \\ \lambda\left(Q_{\bar{r}}\right) \subset \mathcal{C}_{f / k}, & e^{k h Q_{r}}=I\end{cases}
$$

it is said that $\Phi(t, 0)=P(t, 0) e^{Q t}$ is reducible; if (21) holds only if $Q_{r}=0$, it is said that $\Phi(t, 0)=P(t, 0) e^{Q t}$ is irreducible. $e^{Q_{r} t}$ is called a reducing factor.

(iii) Let $\Phi(t, 0)=P(t, 0) e^{Q t}$ be a kh-periodic $\mathcal{C}$ - (resp., $\mathcal{J}$ - and $\mathcal{H}$-) Floquet factorization. If $Q$ is fixed under principal logarithms branch specifications, $\Phi(t, 0)=P(t, 0) e^{Q t}$ is called a principal $\mathcal{C}$ - (resp., $\mathcal{J}$ - and $\mathcal{H}$-) Floquet factorization; otherwise, it is non-principal.

Remark 12 Reducibility means that a redundant factor appears in $e^{Q t}$ and $P(t, 0)$, if they are treated separately. Such a reducing factor cancels each other in $P(t, 0) e^{Q t}$. In other words, reducible Floquet factorizations may 'distort' structure of the FDLCP system, when $e^{Q t}$ and $P(t, 0)$ are employed separately. Reducible Floquet factorization can be brought in by non-principal logarithm branches as will be seen soon.

Remark 13 The last relation of (21) yields that $e^{Q_{r}(t+k h)}=e^{Q_{r} t}$ for all $t$. A kh-periodic Floquet factorization is irreducible, it must be a kh-periodic Floquet simplex. After confirming the commutativity between $Q_{r}$ and $Q_{\bar{r}}$, we can prove that the reverse assertion is also true if some eigenvalue assumption on $Q$ is satisfied. We emphasize here that simplicity and irreducibility of Floquet factorizations reflect different aspects of Floquet factorizations.

\subsection{Commutativity of Floquet Factorizations}

The FDLCP system (10) is monodromy commutative if it holds

$$
A(t) \Phi(h, 0)=\Phi(h, 0) A(t), \quad \forall t \in[0, h)
$$

By the $h$-periodicity of $A(t),(22)$ can be extended to the whole interval $J$. An LTI system must be monodromy commutative. A proof for Theorem 12 is given in Appendix E.

Theorem 12 In the FDLCP system (10), assume that $A(t) \in \mathcal{F}^{n \times n}$ is locally integrable and monodromy commutative on $J$. Then the kh-periodic $\mathcal{C}$ - and $\mathcal{H}$-Floquet factorization of $\Phi(t, 0)$ of Theorem 1 are commutative; that is, $\Phi(t, 0)=P(t, 0) e^{Q t}=e^{Q t} P(t, 0)$ for all $t \in J$, where $P(t, 0)$ is absolutely continuous and nonsingular for each $t \in J$.

Remark 14 Since $\Phi(t, \tau)=\Phi(t, 0) \Phi^{-1}(\tau, 0)$ over $t, \tau \in J$, there exists a kh-periodic Floquet factorization $\Phi(t, \tau)=P(t, 0) P^{-1}(\tau, 0) e^{Q(t-\tau)}$ in a monodromy commutative system. $P(t, 0) P^{-1}(\tau, 0)$ is kh-periodic both in $t$ and $\tau$, absolutely continuous and nonsingular in $t$ and $\tau$.

Theorem 12 also applies to the $\mathcal{C}$-and $\mathcal{H}$-Floquet factorizations of Theorems 2 and 3. However, commutativity of $\mathcal{J}$-Floquet factorizations cannot be guaranteed in general, which is due to similarity transformations in Jordan canonical forms that are not unique. 


\subsection{Simplicity of Floquet Factorizations}

Based on Theorem 10, it is straightforward to show the following theorem about Floquet simplices, as far as the Floquet factorizations defined in Theorem 1 are concerned. Simplicity of Floquet factorizations other than those defined in Theorem 1 can be also examined similarly.

Theorem 13 In the FDLCP system (10), assume that $A(t) \in \mathcal{F}^{n \times n}$ is locally integrable on $J$.

(i) If $\Phi(t, 0)=P(t, 0) e^{Q t}$ is the principal kh-periodic $\mathcal{C}$ - (resp., $\mathcal{J}$-) Floquet factorization of Theorem 1, then $\Phi(t, 0)=P(t, 0) e^{Q t}$ is a kh-periodic Floquet simplex. Moreover, any nonprincipal kh-periodic $\mathcal{C}$ - (resp., $\mathcal{J}$-) Floquet factorization is not a Floquet simplex;

(ii) Let $\Phi(t, 0)=P(t, 0) e^{Q t}$ be a kh-periodic $\mathcal{H}$-Floquet factorization of Theorem 1. $\Phi(t, 0)=$ $P(t, 0) e^{Q t}$ is a kh-periodic Floquet simplex if and only if for any eigenvalue $\lambda_{i}(\Phi(h, 0))$ of the monodromy, it holds that $(k h)^{-1} H\left(\lambda_{i}^{k}(\Phi(h, 0)) \in \mathcal{C}_{f / k}\right.$.

Proof of Theorem 13 For brevity, we only show the case of $\mathcal{C}$-Floquet factorizations. By the first assertion in Theorem 10, we have $\lambda_{i}(Q)=(k h)^{-1} \ln \left(p, \lambda_{i}^{k}(\Phi(h, 0))\right)$. Note by definition that

$$
\ln \left(p, \lambda_{i}^{k}(\Phi(h, 0))\right)=\operatorname{Ln}\left(\left|\lambda_{i}^{k}(\Phi(h, 0))\right|\right)+j\left(\arg \left(\lambda_{i}^{k}(\Phi(h, 0))+2 \pi p\right)\right.
$$

This, together with $-\pi<\arg (\cdot) \leq \pi$, means that if $p=0$, then $-\omega_{k h} / 2<(k h)^{-1} \arg \left(\lambda_{i}^{k}(\Phi(h, 0)) \leq\right.$ $\omega_{k h} / 2$ and thus $\lambda_{i}(Q) \in \mathcal{C}_{f / k}$; on the other hand, for any $p \neq 0$, it cannot be true that $-\omega_{k h} / 2<$ $(k h)^{-1} \arg \left(\lambda_{i}^{k}(\Phi(h, 0)) \leq \omega_{k h} / 2\right.$, thus $\lambda_{i}(Q) \notin \mathcal{C}_{f / k}$.

Remark 15 By Theorem 13, we can obtain Floquet simplices by specifying principal logarithm branches in the Cauchy integration formula and Jordan canonical forms of Theorem 1. However, to get a Floquet simplex through Hermite interpolation polynomials may need extra works, even if principal logarithm branches are specified.

\subsection{Reducibility of Floquet Factorizations}

Theorem 14 Assume in (10) that $A(t) \in \mathcal{F}^{n \times n}$ is locally integrable on $J$. Let $\Phi(t, 0)=P(t, 0) e^{Q t}$ be a kh-periodic Floquet factorization. $\Phi(t, 0)=P(t, 0) e^{Q t}$ is reducible if and only if there are $Q_{r} \neq 0$ and $Q_{\bar{r}} \neq 0$ such that $Q=Q_{r}+Q_{\bar{r}}, e^{k h Q_{r}}=I, \lambda\left(Q_{\bar{r}}\right) \subset \mathcal{C}_{f / k}$, and $Q_{r} Q_{\bar{r}}=Q_{\bar{r}} Q_{r}$.

Proof of Theorem 14 By Definition 3, it remains to show commutativity between $Q_{r}$ and $Q_{\bar{r}}$. To see the necessity, assume that $\Phi(t, 0)=P(t, 0) e^{Q t}$ is reducible. We recall (20) and observe

$$
\begin{aligned}
e^{-Q_{r} t} Q e^{Q_{r} t} & =e^{-Q_{r} t} P^{-1}(t, 0) A(t) P(t, 0) e^{Q_{r} t}-e^{-Q_{r} t} P^{-1}(t, 0) \dot{P}(t, 0) e^{Q_{r} t} \\
& =\tilde{P}^{-1}(t, 0) A(t) \tilde{P}(t, 0)-\tilde{P}^{-1}(t, 0) \dot{P}(t, 0) e^{Q_{r} t}
\end{aligned}
$$

where $\tilde{P}(t, 0)=P(t, 0) e^{Q_{r} t}$. Apparently, $\Phi(t, 0)=\tilde{P}(t, 0) e^{Q_{\bar{r}} t}$ is also a $k h$-Floquet factorization. Thus (20) holds in term of $\Phi(t, 0)=\tilde{P}(t, 0) e^{Q_{\bar{r}}} t$; that is,

$$
Q_{\bar{r}}=\tilde{P}^{-1}(t, 0) A(t) \tilde{P}(t, 0)-\tilde{P}^{-1}(t, 0) \dot{\tilde{P}}(t, 0)
$$

Combining the above equations, we are led that

$$
\begin{aligned}
& e^{-Q_{r} t} Q e^{Q_{r} t}-Q_{\bar{r}}=-\tilde{P}^{-1}(t, 0)\left(\dot{P}(t, 0) e^{Q_{r} t}-\dot{\tilde{P}}(t, 0)\right) \\
= & -\tilde{P}^{-1}(t, 0)\left(\dot{P}(t, 0) e^{Q_{r} t}-\dot{P}(t, 0) e^{Q_{r} t}-P(t, 0) e^{Q_{r} t} Q_{r}\right) \\
= & \tilde{P}^{-1}(t, 0) P(t, 0) e^{Q_{r} t} Q_{r}=e^{-Q_{r} t} P^{-1}(t, 0) P(t, 0) e^{Q_{r} t} Q_{r}=Q_{r}
\end{aligned}
$$


which implies in particular that $e^{-Q_{r} t} Q_{\bar{r}} e^{Q_{r} t}=Q_{\bar{r}}$; or equivalently, $Q_{\bar{r}} e^{Q_{r} t}=e^{Q_{r} t} Q_{\bar{r}}$, whose derivative with respect to $t$ leads readily that $Q_{r} Q_{\bar{r}}=Q_{\bar{r}} Q_{r}$.

To see the sufficiency, we note that $Q_{r} Q_{\bar{r}}=Q_{\bar{r}} Q_{r}$ means $e^{Q t}=e^{\left(Q_{r}+Q_{\bar{r}}\right) t}=e^{Q_{r} t} e^{Q_{\bar{r}} t}$. This, together with the assumptions on $Q_{r}$ and $Q_{\bar{r}}$, says that $\Phi(t, 0)=P(t, 0) e^{Q t}$ is reducible.

By use of Theorem 9, we consider reducibility of $k h$-periodic $\mathcal{C}$-, $\mathcal{J}$ - and $\mathcal{H}$-Floquet factorizations obtained through the algorithms in Theorem 1. Theorem 15 is proved in Appendix F.

Theorem 15 In the FDLCP system (10), let $A(t) \in \mathcal{F}^{n \times n}$ be locally integrable. Then

(i) Any non-principal kh-periodic $\mathcal{C}$ - and $\mathcal{J}$-Floquet factorizations of Theorem 1 are reducible;

(ii) Let $\Phi(t, 0)=P(t, 0) e^{Q t}$ be a kh-periodic $\mathcal{H}$-Floquet factorization of Theorem 1. If $\Phi(t, 0)=$ $P(t, 0) e^{Q t}$ is irreducible, then any kh-periodic $\mathcal{H}$-Floquet factorizations of Theorem 1 with logarithm branches other than those in $\Phi(t, 0)=P(t, 0) e^{Q t}$ are reducible.

\subsection{Equivalence between Simplicity and Irreducibility}

Now we see that a Floquet simplex is irreducible under some eigenvalue conditions about $Q$. Therefore, in most FDLCP systems an irreducible $k h$-periodic Floquet factorization can be obtained by determining a $k h$-periodic Floquet simplex, as suggested in Theorems 4,5 and 6 . A proof for Theorem 16 is given in Appendix G.

Theorem 16 In the FDLCP system (10), assume that $A(t) \in \mathcal{F}^{n \times n}$ is locally integrable on $J$. Let $\Phi(t, 0)=P(t, 0) e^{Q t}$ be a kh-periodic Floquet factorization. For any distinct eigenvalue of $Q$, there is only one corresponding Jordan block. Then $\Phi(t, 0)=P(t, 0) e^{Q t}$ is a kh-periodic Floquet simplex if and only if $\Phi(t, 0)=P(t, 0) e^{Q t}$ is irreducible.

\section{Conclusion}

Floquet theory plays an irreplaceable role in analysis and synthesis problems of FDLCP control systems. In this paper, we first concentrate our attention on collecting and reviewing basic facts about Floquet factorizations and their derivations. Based on these facts, we turn to establish a more general framework for classifying Floquet factorizations, while significant characteristics about the Floquet factorizations are claimed and proved rigorously. More precisely, the following aspects are considered: (i) Floquet factorization algorithms; (ii) Properties of Floquet factors; (iii) Relationships among Floquet factorizations.

\section{Appendix A: Proof of Lemma 3}

We modify the arguments of Lemma 7.1 [13]. First we take $\eta>0$ such that $\eta \sigma_{\min }(C)>1$. Here $\sigma_{\min }(\cdot)$ denotes the minimum singular value. Such $\eta$ always exists. The aim to introduce $\eta$ will be understood in the later arguments. We will see that $\eta$ can be removed eventually.

For a nonsingular matrix $T, T^{-1}(\eta C) T=e^{T^{-1} B T}$ as long as $\eta C=e^{B}$. Without loss of generality, let $\eta C$ be in the Jordan canonical form (4) with a single block, say $\eta C=J_{i}$. Define

$$
B=: \ln \left(p_{i}, \lambda_{i}\right) I_{i}+\ln \left(p_{i}, I_{i}+\lambda_{i}^{-1} E_{i}\right), \quad p_{i} \in \mathcal{Z}
$$

we show that $B$ is a well-defined matrix logarithm in the sense that $J_{i}=e^{B}$. 
To this end, we compute $\ln \left(p_{i}, I_{i}+\lambda_{i}^{-1} E_{i}\right)$ in (23). Expand $\ln (1+z)$ into its Taylor series in the open disc $\{z:|z|<1\}$ by Theorem 5.1 and Example 5.2 [29, pp.78-79]. We obtain

$$
\ln (1+z)=\sum_{l=1}^{\infty} l^{-1}(-1)^{l+1} z^{l}, \quad \forall|z|<1
$$

from which the $p_{i}$-th logarithm branch of (1) and (2) can be expressed as follows.

$$
\ln \left(p_{i}, 1+z\right)=\sum_{l=1}^{\infty} l^{-1}(-1)^{l+1} z^{l}+j 2 \pi p_{i}, \quad \forall|z|<1
$$

Note that $\left\|E_{i} / \lambda_{i}\right\|=\left|\lambda_{i}\right|^{-1}|| E_{i} \|=\left|\lambda_{i}\right|^{-1} \leq \sigma_{\min }^{-1}(\eta C)<1$. It is validated to substitute $E_{i} / \lambda_{i}$ for $z$ in (24). In other words, the factor $\eta$ guarantees $\left\|E_{i} / \lambda_{i}\right\|<1$; otherwise, the series of $\ln \left(p_{i}, I_{i}+E_{i} / \lambda_{i}\right)$ may be invalid. This point seems to be neglected in the proof for Lemma 7.1 of [13] and the arguments in [4, pp.65-67] and Theorem 6.1 of [14, pp.60-62].

Substituting $E_{i} / \lambda_{i}$ for $z$ in (24) and noting that $E_{i}^{l}=0$ for any $l \geq m_{i}$, we obtain

$$
\begin{aligned}
& \ln \left(p_{i}, I_{i}+\lambda_{i}^{-1} E_{i}\right)=\sum_{l=1}^{\infty} \frac{(-1)^{l+1}}{l} \frac{E_{i}^{l}}{\lambda_{i}^{l}}+j 2 \pi p_{i} I_{i} \\
= & \sum_{l=1}^{m_{i}-1} \frac{(-1)^{l+1}}{l} \frac{E_{i}^{l}}{\lambda_{i}^{l}}+j 2 \pi p_{i} I_{i}=\ln \left(I_{i}+\lambda_{i}^{-1} E_{i}\right)+j 2 \pi p_{i} I_{i}
\end{aligned}
$$

Based on (25) and the specific definition of $B$, let us observe that

$$
\begin{aligned}
& e^{B}=\exp \left\{\ln \left(p_{i}, \lambda_{i}\right) I_{i}+\ln \left(p_{i}, I_{i}+\lambda_{i}^{-1} E_{i}\right)\right\} \\
= & \exp \left\{\ln \left(p_{i}, \lambda_{i}\right) I_{i}\right\} \exp \left\{\ln \left(p_{i}, I_{i}+\lambda_{i}^{-1} E_{i}\right)\right\} \\
= & \exp \left\{\ln \left(\lambda_{i}\right) I_{i}+j 2 \pi p_{i} I_{i}\right\} \exp \left\{\ln \left(I_{i}+\lambda_{i}^{-1} E_{i}\right)+j 2 \pi p_{i} I_{i}\right\} \\
= & \exp \left\{\ln \left(\lambda_{i}\right) I_{i}\right\} \exp \left\{\ln \left(I_{i}+\lambda_{i}^{-1} E_{i}\right)\right\}=\lambda_{i} I_{i}\left(I_{i}+\lambda_{i}^{-1} E_{i}\right)=J_{i}
\end{aligned}
$$

where we noticed that $\ln \left(p_{i}, \lambda_{i}\right) I_{i}$ and $\ln \left(p_{i}, I_{i}+E_{i} / \lambda_{i}\right)$ are commutative and $e^{\ln (z)}=z$ for any $z$ [29]. This leads that $\eta C=e^{B}$. Taking into account $\eta=e^{\ln (\eta)}$, it follows

$$
C=e^{B-\ln (\eta) I}=: e^{\tilde{B}}
$$

Now let us define $\ln \left(p_{i}, I_{i}+E_{i} / \lambda_{i}\right)$ by (25) even if $\left\|E_{i} / \lambda_{i}\right\| \geq 1$. Clearly, this definition itself makes sense since there are only finitely many non-zero terms in (25). Therefore, the matrix $B$ in (23) is well-defined and the arguments in (26) are validated even if $\left\|E_{i} / \lambda_{i}\right\| \geq 1$.

Furthermore, $e^{\tilde{B}}$ is analytic with respect to $\tilde{B}$ as long as $\tilde{B}$ is bounded. The analytic continuation theory [29, pp.89-91] says that (27) holds ture for both $\left\|E_{i} / \lambda_{i}\right\| \geq 1$ and $\left\|E_{i} / \lambda_{i}\right\|<1$. In short, the assertion $C=e^{B}$ follows by letting $\eta=1$ in (27).

If $C$ is real, the matrix $B$ is given by (6). This can be proved by replacing $\ln \left(p_{i}, \lambda_{i}\right) I_{i}$ and $E_{i} / \lambda_{i}$ with $\ln \left(p_{i}, \Lambda_{i}\right)$ and $\Lambda_{i}^{-1} F_{i}$, respectively, in the above. Clearly, $\ln \left(p_{i}, \Lambda_{i}\right) \Lambda_{i}^{-1}=\Lambda_{i}^{-1} \ln \left(p_{i}, \Lambda_{i}\right)$ and $\Lambda_{i}^{-1} F_{i}=F_{i} \Lambda_{i}$, which can be shown by computations. Then, $\bar{B} B=B \bar{B}$ follows since $B$ is blockwise diagonal and $T$ is real. Finally, $C^{2}=C \bar{C}=e^{B \overline{\left(e^{B}\right)}}=e^{B} e^{\bar{B}}=e^{B+\bar{B}}$.

\section{Appendix B: Proof of Theorem 1}

We only prove the case for the $k h$-periodic $\mathcal{C}$-Floquet factorization. By Theorem 6.3.2 [19], $\Phi(t, 0)$ is well-defined, invertible and absolutely continuous for all $t \in J$. Lemma 1 and the proof about Lemma 8.1.1 [19] tell that there is a (possibly complex) constant matrix

$$
Q=\frac{1}{2 k h \pi j} \oint_{\partial \Omega} \ln (p, z)[z I-\Phi(k h, 0)]^{-1} d z
$$


such that $\Phi(k h, 0)=e^{Q k h}$. To see this, we observe from the $h$-periodicity of $A(t)$ that

$$
\begin{aligned}
& \Phi(t+k h, k h) \\
= & I+\int_{k h}^{t+k h} A(\tau) d \tau+\int_{k h}^{t+k h}\left[\int_{\tau_{1}}^{t+k h} A\left(\tau_{2}\right) d \tau_{2}\right] A\left(\tau_{1}\right) d \tau_{1}+\cdots \\
= & I+\int_{0}^{t} A(\tilde{\tau}+k h) d \tilde{\tau}+\int_{0}^{t}\left[\int_{\tilde{\tau}_{1}+k h}^{t+k h} A\left(\tau_{2}\right) d \tau_{2}\right] A\left(\tilde{\tau}_{1}+k h\right) d \tilde{\tau}_{1}+\cdots \\
= & I+\int_{0}^{t} A(\tilde{\tau}+k h) d \tilde{\tau}+\int_{0}^{t}\left[\int_{\tilde{\tau}_{1}}^{t} A\left(\tilde{\tau}_{2}+k h\right) d \tilde{\tau}_{2}\right] A\left(\tilde{\tau}_{1}+k h\right) d \tilde{\tau}_{1}+\cdots \\
= & I+\int_{0}^{t} A(\tilde{\tau}) d \tilde{\tau}+\int_{0}^{t}\left[\int_{\tilde{\tau}_{1}}^{t} A\left(\tilde{\tau}_{2}\right) d \tilde{\tau}_{2}\right] A\left(\tilde{\tau}_{1}\right) d \tilde{\tau}_{1}+\cdots=\Phi(t, 0)
\end{aligned}
$$

where $\tilde{\tau}+k h=\tau, \tilde{\tau}_{1}+k h=\tau_{1}, \tilde{\tau}_{2}+k h=\tau_{2}$ and so on. It follows by (29) that

$$
\Phi(k h, 0)=\Phi(k h,(k-1) h) \Phi((k-1) k,(k-2) h) \cdots \Phi(h, 0)=\Phi^{k}(h, 0)
$$

Using this in (28) leads $\Phi(k h, 0)=e^{Q k h}$. Denote $P(t, 0)=\Phi(t, 0) e^{-Q t}$. Based on (29), we have

$$
\begin{aligned}
P(t+k h, 0) & =\Phi(t+k h, 0) e^{-Q(t+k h)}=\Phi(t+k h, k h) \Phi(k h, 0) e^{-Q(t+k h)} \\
& =\Phi(t, 0) e^{Q k h} e^{-Q(t+k h)}=\Phi(t, 0) e^{-Q t}=P(t, 0)
\end{aligned}
$$

Thus, $P(t, 0)$ is $k h$-periodic. $P(t, 0)$ is nonsingular since $\Phi(t, 0)$ and $e^{-Q t}$ are nonsingular. Absolute continuity of $P(t, 0)$ comes from that of $\Phi(t, 0)$ and $e^{-Q t}$. Clearly, $e^{-Q t}$ is absolute continuous since $e^{-Q t}$ is continuously differentiable. Since $\Phi(0,0)=I, P(0,0)=I$ is evident.

Asymptotic stability follows from the LTI system $\dot{\tilde{x}}=Q \tilde{x}$ after the state transform $\tilde{x}=P(t, 0) x$ in (10). Asymptotic stability via the eigenvalues of $\Phi(h, 0)$ can be shown with (11).

\section{Appendix C: Proof of Theorem 9}

To show the assertion (i), we note by the Cauchy integration formula that

$$
\begin{aligned}
Q_{1}-Q_{2} & =\frac{1}{2 k h \pi j} \oint_{\partial \Omega}\left(\ln \left(p_{1}, z\right)-\ln \left(p_{2}, z\right)\right)\left[z I-\Phi^{k}(h, 0)\right]^{-1} d z \\
& =\frac{1}{2 k h \pi j} \oint_{\partial \Omega}\left(\ln (z)+j 2 \pi p_{1}-\ln (z)-j 2 \pi p_{2}\right)\left[z I-\Phi^{k}(h, 0)\right]^{-1} d z \\
& =\frac{2 \pi\left(p_{1}-p_{2}\right) j}{2 k h \pi j} \oint_{\partial \Omega}\left[z I-\Phi^{k}(h, 0)\right]^{-1} d z
\end{aligned}
$$

The assertion follows if we note that $(2 \pi j)^{-1} \oint_{\partial \Omega}\left[z I-\Phi^{k}(h, 0)\right]^{-1} d z=I$ by Theorem 7.5 .1 of [19] since all the singular points of $\left[z I-\Phi^{k}(h, 0)\right]^{-1}$ are in the interior of $\Omega$. Clearly, $Q_{1} \neq Q_{2}$ as long as $p_{1} \neq p_{2}$ by the above equation.

To show the assertion (ii), we note by (5) that

$$
Q_{1}-Q_{2}=(k h)^{-1} T^{-1} \operatorname{diag}\left[\left(\ln \left(p_{1}, \lambda_{1}\right)-\ln \left(p_{1}^{\prime}, \lambda_{1}\right)\right) I_{1}, \cdots,\left(\ln \left(p_{\alpha}, \lambda_{\alpha}\right)-\ln \left(p_{\alpha}^{\prime}, \lambda_{\alpha}\right)\right) I_{\alpha}\right] T
$$

The assertion follows if we note that $\ln \left(p_{i}, \lambda_{i}\right)-\ln \left(p_{i}^{\prime}, \lambda_{i}\right)=j 2 \pi\left(p_{i}-p_{i}^{\prime}\right)_{m_{i}}$. Under different logarithm branch specifications, $Q_{1} \neq Q_{2}$ is obvious.

To see the assertion (iii), we recall (7). The Hermite interpolation polynomial for $Q_{1}$ is determined when the vector in the right-hand side of (7) is used, while the Hermite interpolation 
polynomial for $Q_{2}$ is determined when the vector in the right-hand side of (7) is replaced with

$$
\left[\begin{array}{c}
\vdots \\
\hline \ln ^{\left(m_{i}-1\right)}\left(p_{i}^{\prime}, \lambda_{i}\right) \\
\vdots \\
\ln ^{(1)}\left(p_{i}^{\prime}, \lambda_{i}\right) \\
\hline \ln ^{(0)}\left(p_{i}^{\prime}, \lambda_{i}\right) \\
\hline \vdots
\end{array}\right]=\left[\begin{array}{c}
\vdots \\
\frac{\ln ^{\left(m_{i}-1\right)}\left(p_{i}, \lambda_{i}\right)}{\vdots} \\
\frac{\ln (1)}{\ln \left(p_{i}, \lambda_{i}\right)} \\
\vdots \vdots
\end{array}\right]-\left[\begin{array}{c}
\vdots \\
0 \\
\vdots \\
0 \\
\frac{j 2 \pi\left(p_{i}-p_{i}^{\prime}\right)}{\vdots}
\end{array}\right]
$$

Here we used the facts that $\ln ^{(0)}\left(p_{i}^{\prime}, \lambda_{i}\right)=\ln \left(p_{i}^{\prime}, \lambda_{i}\right)=\ln ^{(0)}\left(\lambda_{i}\right)+j 2 \pi p_{i}^{\prime}$ and that for any $l \geq 1$, $\ln ^{(l)}\left(p_{i}^{\prime}, \lambda_{i}\right)=\ln ^{(l)}\left(\lambda_{i}\right)=\ln ^{(l)}\left(p_{i}, \lambda_{i}\right)$. The latter can be shown by (1). It follows that

$$
Q_{1}-Q_{2}=\sum_{i=0}^{m-1} h_{i}\left(\Phi^{k}(h, 0)\right)^{i}-\sum_{i=0}^{m-1}\left(h_{i}-\xi_{i}\right)\left(\Phi^{k}(h, 0)\right)^{i}
$$

Also related to the assertion (iii), to see $Q_{1} \neq Q_{2}$ under different logarithm branches, we assume $Q_{1}=Q_{2}$. It follows that $H_{1}\left(\Phi^{k}(h, 0)\right)=Q_{1}=Q_{2}=H_{2}\left(\Phi^{k}(h, 0)\right)$, where $H_{1}(s)$ and $H_{2}(s)$ are the Hermite interpolation polynomials for $\Phi(t, 0)=P_{1}(t, 0) e^{Q_{1} t}$ and $\Phi(t, 0)=P_{2}(t, 0) e^{Q_{2} t}$, respectively. Since $H_{1}(s)$ and $H_{2}(s)$ are unique, we are led to a contradiction.

To see the last assertion, we note by direct observation that $Q_{1}$ and $Q_{2}$ are commutative in each case. Then, $P_{1}(t, 0)=\Phi(t, 0) e^{-Q_{1} t}=\Phi(t, 0) e^{-Q_{2} t} e^{\left(Q_{2}-Q_{1}\right) t}=P_{2}(t, 0) e^{\left(Q_{2}-Q_{1}\right) t}$.

\section{Appendix D: Proof of Theorem 10}

By Property 1.7(e) of $[2, \mathrm{p} .16]$, we have $\operatorname{det}\left(e^{A}\right)=e^{\operatorname{tr}(A)}$. Recalling properties about the determinant and trace, the result (16) follows by (29) and Definition 1 that $\Phi^{k}(h, 0)=e^{k h Q}$.

To show the assertion (i), let us express the monodromy matrix $\Phi(h, 0)$ by its Jordan canonical form, say $\Phi(h, 0)=T_{\Phi}^{-1} J_{\Phi(h, 0)} T_{\Phi}$. We note by definition that

$$
\begin{aligned}
& Q=\frac{1}{2 k h \pi j} \oint_{\partial \Omega} \ln (p, z) T_{\Phi}^{-1}\left[z I-J_{\Phi(h, 0)}^{k}\right]^{-1} T_{\Phi} d z \\
= & \frac{1}{2 k h \pi j} T_{\Phi}^{-1} \oint_{\partial \Omega} \ln (p, z)\left[z I-J_{\Phi(h, 0)}^{k}\right]^{-1} d z T_{\Phi} \\
= & \frac{1}{2 k h \pi j} T_{\Phi}^{-1} \oint_{\partial \Omega} \ln (p, z)\left(\operatorname{diag}\left[\cdots, z-\lambda_{i}^{k}(\Phi(h, 0)), \cdots\right]+[*]\right)^{-1} d z T_{\Phi} \\
= & \frac{1}{k h} T_{\Phi}^{-1}\left\{\operatorname{diag}\left[\cdots, \frac{1}{2 \pi j} \oint_{\partial \Omega} \ln (p, z)\left(z-\lambda_{i}^{k}(\Phi(h, 0))\right)^{-1}, \cdots\right]+[*]\right\} d z T_{\Phi} \\
= & \frac{1}{k h} T_{\Phi}^{-1}\left\{\operatorname{diag}\left[\cdots, \ln \left(p, \lambda_{i}^{k}(\Phi(h, 0))\right), \cdots\right]+[*]\right\} d z T_{\Phi}
\end{aligned}
$$

where $[*]$ is an upper trianglar matrix with zero diagonal entries whose exact expressions are not needed. The fourth equation of (30) follows since $J_{\Phi(h, 0)}^{k}$ is upper trianglar and the diagonal entries of the corresponding inverse matrix are the reciprocals of the diagonal entries. The last equation of (30) follows by the theorem of residue [29].

To see the assertion (ii), let us observe from Lemma 3 and Theorem 1 that

$$
Q=\frac{1}{k h} T^{-1} \operatorname{diag}\left[\cdots, \ln \left(p_{i}, \lambda_{i}^{k}(\Phi(h, 0))\right) I_{i}-\sum_{l=1}^{m_{i}-1} \frac{\left(-E_{i}\right)^{l}}{l\left(\lambda_{i}^{k}(\Phi(h, 0))\right)^{l}}, \cdots\right] T
$$

where $E_{i}$ is nilpotent and upper trianglar and thus all diagonal entries of the upper trianglar matrix $\left(-E_{i}\right)^{l} / l\left(\lambda_{i}^{k}(\Phi(h, 0))\right)^{l}$ are zeros for each $l$. 
The assertion (iii) is obvious by the following observation about matrix polynomials.

$$
\begin{aligned}
Q & =(k h)^{-1} H\left(\Phi^{k}(h, 0)\right)=(k h)^{-1} H\left(T_{\Phi}^{-1} J_{\Phi(h, 0)}^{k} T_{\Phi}\right) \\
& =(k h)^{-1} T_{\Phi}^{-1}\left(\operatorname{diag}\left[\cdots, H\left(\lambda_{i}^{k}(\Phi(h, 0))\right), \cdots\right]+[*]\right) T_{\Phi}
\end{aligned}
$$

where $[*]$ is defined in a way similar to that in $(30)$.

\section{Appendix E: Proof of Theorem 12}

Theorem 1 says that the $k h$-periodic $\mathcal{C}$ - (resp., $\mathcal{H}$-)Floquet factorization $\Phi(t, 0)=P(t, 0) e^{Q t}$ possesses $P(t, 0)$ and $Q$ with the desired features. It suffices to show that $\Phi(t, 0) e^{Q t}=e^{Q t} \Phi(t, 0)$ for all $t$. Since $e^{Q t}=\sum_{k=0}^{\infty}(i !)^{-1} Q^{i} t^{i}$, thus $\Phi(t, 0) e^{Q t}=e^{Q t} \Phi(t, 0)$ will be meant if we have

$$
\Phi(t, 0) Q=Q \Phi(t, 0), \quad \forall t \in J
$$

Firstly, we show that $(31)$ is true for the $\mathcal{C}$-Floquet factorization case. Since the Cauchy integration formula is independent of the path $\partial \Omega$, we introduce a specific integral path [15].

By the assumption on $A(t), \Phi(t, 0)$ is continuous. We have: (i) $\Phi^{k}(h, 0)$ is nonsingular; (ii) for some $K>0,\left\|\Phi^{k}(h, 0)\right\| \leq K$; (iii) $\Phi^{k}(h, 0)$ has at most $n$ distinct eigenvalues. By (i), $\lambda=0$ is not an eigenvalue of $\Phi^{k}(h, 0)$. By (ii) and (iii), we can find $R>\max \{1, K\}$ and $\theta \in[0,2 \pi)$ such that all eigenvalues of $\Phi^{k}(h, 0)$ lay in a simply connected region $D_{R, \theta}$. Here, $D_{R, \theta}$ denotes the open region between the circles $C_{R}:\{z:|z|=R\}$ and $C_{R^{-1}}:\left\{z:|z|=R^{-1}\right\}$, excluding the ray segment $S_{R}=:\left\{z=r e^{j \theta}: R^{-1} \leq r \leq R\right\}$. Correspondingly, the boundary $\partial D_{R, \theta}$ satisfies

$$
\partial D_{R, \theta}=C_{R} \cup S_{R}^{(r)} \cup C_{R^{-1}} \cup S_{R}^{(l)}
$$

where $C_{R}$ and $C_{R^{-1}}$ are oriented in the positive direction, while $S_{R}^{(l)}$ and $S_{R}^{(r)}$, respectively, denote the left- and right-hand sides of $S_{R}$ in the positive direction sense of $\partial D_{R, \theta}$.

Then, the Cauchy integration formula (3) yields

$$
\begin{aligned}
Q & =\frac{1}{2 k h \pi j} \oint_{\partial D_{R, \theta}} \ln (p, z)\left[z I-\Phi^{k}(h, 0)\right]^{-1} d z \\
& =\frac{1}{2 k h \pi j}\left(\oint_{C_{R}}+\oint_{C_{R^{-1}}}+\oint_{S_{R}^{(r)}}+\oint_{S_{R}^{(l)}}\right) \ln (p, z)\left[z I-\Phi^{k}(h, 0)\right]^{-1} d z \\
& =\frac{1}{2 k h \pi j}\left(\oint_{C_{R}}+\oint_{C_{R^{-1}}}\right) \ln (p, z)\left[z I-\Phi^{k}(h, 0)\right]^{-1} d z
\end{aligned}
$$

in which we note that $\int_{S_{R}^{(r)}}$ is just negative to $\int_{S_{R}^{(l)}}$.

On the one hand, $\left\|\Phi^{k}(h, 0)\right\|<|z|$ for any $z \in C_{R}$, based on which the following expansion about $\left[z I-\Phi^{k}(h, 0)\right]^{-1}$ is validated by Corollary 2 of [?, p.85].

$$
\left[z I-\Phi^{k}(h, 0)\right]^{-1}=z^{-1} \sum_{i=0}^{\infty}\left(\Phi^{k}(h, 0)\right)^{i} z^{-i}, \quad \forall z \in C_{R}
$$

By the $h$-periodicity of $A(t)$ and its monodromy commutativity $(22)$, the definition of $\Phi(t, 0)$ (see (29) for reference) says that $\Phi(t, 0) \Phi^{k}(h, 0)=\Phi^{k}(h, 0) \Phi(t, 0)$ for all $t \in J$. We have by (34) that

$$
\begin{aligned}
& \Phi(t, 0) \frac{1}{2 k h \pi j} \oint_{C_{R}} \ln (p, z)\left[z I-\Phi^{k}(h, 0)\right]^{-1} d z \\
= & \frac{1}{2 k h \pi j} \oint_{C_{R}} \ln (p, z) \frac{1}{z} \sum_{i=0}^{\infty} \Phi(t, 0)\left(\Phi^{k}(h, 0)\right)^{i} \frac{1}{z^{i}} d z \\
= & \frac{1}{2 k h \pi j} \oint_{C_{R}} \ln (p, z) \frac{1}{z} \sum_{i=0}^{\infty}\left(\Phi^{k}(h, 0)\right)^{i} \frac{1}{z^{i}} d z \Phi(t, 0) \\
= & \frac{1}{2 k h \pi j} \oint_{C_{R}} \ln (p, z)\left[z I-\Phi^{k}(h, 0)\right]^{-1} d z \Phi(t, 0)
\end{aligned}
$$


On the other hand, $|z| \cdot|| \Phi^{-k}(h, 0) \|<1$ for any $z \in C_{R^{-1}}$, based on which the following series expansion about $\left[z \Phi^{k}(h, 0)-I\right]^{-1}$ is validated by Theorem 2 of [?, p.83].

$$
\left[z \Phi^{-k}(h, 0)-I\right]^{-1}=-\sum_{i=0}^{\infty}\left(\Phi^{-k}(h, 0)\right)^{i} z^{i}, \quad \forall z \in C_{R^{-1}}
$$

It is easy to see that $\Phi(t, 0) \Phi^{-k}(h, 0)=\Phi^{-k}(h, 0) \Phi(t, 0)$ for all $t \in J$. We obtain by (36) that

$$
\begin{aligned}
& \Phi(t, 0) \frac{1}{2 k h \pi j} \oint_{C_{R^{-1}}} \ln (p, z)\left[z I-\Phi^{k}(h, 0)\right]^{-1} d z \\
= & \Phi(t, 0) \frac{1}{2 k h \pi j} \oint_{C_{R^{-1}}} \ln (p, z)\left[z \Phi^{-k}(h, 0)-I\right]^{-1} \Phi^{-k}(h, 0) d z \\
= & -\frac{1}{2 k h \pi j} \oint_{C_{R^{-1}}} \ln (p, z) \sum_{i=0}^{\infty} \Phi(t, 0)\left(\Phi^{-k}(h, 0)\right)^{i} z^{i} \Phi^{-k}(h, 0) d z \\
= & -\frac{1}{2 k h \pi j} \oint_{C_{R^{-1}}} \ln (p, z) \sum_{i=0}^{\infty}\left(\Phi^{-k}(h, 0)\right)^{i} z^{i} \Phi^{-k}(h, 0) d z \Phi(t, 0) \\
= & \frac{1}{2 k h \pi j} \oint_{C_{R^{-1}}} \ln (p, z)\left[z I-\Phi^{k}(h, 0)\right]^{-1} d z \Phi(t, 0)
\end{aligned}
$$

Bearing (35) and (37) in mind, Eq. (33) yields (31) readily.

Secondly, let us show that $(31)$ is also true in an $\mathcal{H}$-Floquet factorization. Note under the monodromy commutativity that $\Phi(t, 0) \Phi(h, 0)=\Phi(h, 0) \Phi(t, 0)$ for any $t$. We observe

$$
\Phi(t, 0) Q=\Phi(t, 0) H\left(\Phi^{k}(h, 0)\right)=H\left(\Phi^{k}(h, 0)\right) \Phi(t, 0)=Q \Phi(t, 0), \quad \forall t \in J
$$

where $H(\cdot)$ is the Hermite interpolation polynomial of (8)

\section{Appendix F: Proof of Theorem 15}

To see the assertion about $\mathcal{C}$-Floquet factorizations, let $\Phi(t, 0)=P_{1}(t, 0) e^{Q_{1} t}$ be the principal $k h$-periodic $\mathcal{C}$-Floquet factorization, and $\Phi(t, 0)=P_{2}(t, 0) e^{Q_{2} t}$ be a non-principal one; that is, $\Phi(t, 0)=P_{2}(t, 0) e^{Q_{2} t}$ is determined by Theorem 1 in the $p$-th logarithm branch sense $(p \neq 0)$. Theorem 9 says that $Q_{2}=Q_{1}+\frac{j 2 \pi p}{k h} I=: Q_{1}+Q_{0}$, where $Q_{0}=j 2 \pi p /(k h) I \neq 0$ and thus $e^{Q_{2} t}=e^{Q_{1} t+Q_{0} t}=e^{Q_{1} t} e^{Q_{0} t}$ since $Q_{1}$ and $Q_{0}$ are commutative. This, together with $\left.e^{Q_{0} t}\right|_{t=k h}=I$, Theorem 13 and Definition 3, implies that $\Phi(t, 0)=P_{2}(t, 0) e^{Q_{2} t}$ is reducible.

The assertions about $\mathcal{J}$ - and $\mathcal{H}$-Floquet factorizations can be shown similarly.

\section{Appendix G: Proof of Theorem 16}

By Definition 3 and Remark 13, the sufficiency is obvious. It remains to show the necessity. We show that under the eigenvalue condition on $Q$, if $\Phi(t, 0)=P(t, 0) e^{Q t}$ is a $k h$-periodic Floquet simplex, then $\Phi(t, 0)=P(t, 0) e^{Q t}$ is irreducible.

To this end, we assume that $\Phi(t, 0)=P(t, 0) e^{Q t}$ is a $k h$-periodic Floquet simplex but reducible. We do the following observations that will produce us a contradiction.

Firstly, Definitions 2 and 3 tell that there are matrices $Q_{r} \neq 0$ and $Q_{\bar{r}} \neq 0$ satisfying

$$
\begin{cases}Q=Q_{\bar{r}}+Q_{r}, & e^{Q t}=e^{Q_{r} t} e^{Q_{\bar{r}} t}, \quad \forall t \in J \\ \lambda\left(Q_{\bar{r}}\right) \subset \mathcal{C}_{f / k}, & \lambda(Q) \subset \mathcal{C}_{f / k}, \quad e^{k h Q_{r}}=I\end{cases}
$$

We assert from (38) that $Q_{r}$ possesses only eigenvalues $j \omega_{k h} \mu_{i}$ of multiplicity one with $\mu_{i}$ being an integer. Moreover, at least one integer $\mu_{i}$ is not zero. To see this, let $J_{r}$ be a Jordan canonical form of $Q_{r}$. Then $e^{k h Q_{r}}=I$ if and only if $e^{k h J_{r}}=I$. If $J_{r}$ is diagonal, i.e., all eigenvalues of $J_{r}$ are of multiplicity one, the desired assertion is obvious. If $J_{r}$ has a Jordan block corresponding to 
an eigenvalue with multiplicity more than one, the expression of $e^{J_{r} t}$ leads that $e^{k h J_{r}}=I$ cannot be true. Since $Q_{r}$ is nonzero, there is at least one eigenvalue $j \omega_{k h} \mu_{i} \neq 0$.

Secondly, note that for a non-singular matrix $U, U^{-1} Q U=U^{-1} Q_{\bar{r}} U+U^{-1} Q_{r} U$. Theorem 14 tells that $Q_{r}$ and $Q_{\bar{r}}$ are commutative, and thus $U^{-1} e^{Q t} U=e^{U^{-1} Q_{r} U t} e^{U^{-1} Q_{\bar{r}} U t}$ for all $t$. Therefore, it brings us no loss of generality to assume that $Q$ is already expressed in a Jordan canonical form, say $Q=\operatorname{diag}\left[J_{1}, J_{2}, \cdots, J_{\alpha}\right]$, where $J_{i}$ is a Jordan block.

Thirdly, let us write $Q_{r}$ and $Q_{\bar{r}}$ in a blockwise form according to $Q$.

$$
Q_{r}=\left[\begin{array}{ccc}
Q_{r 11} & \cdots & Q_{r 1 \alpha} \\
\vdots & \ddots & \vdots \\
Q_{r \alpha 1} & \cdots & Q_{r \alpha \alpha}
\end{array}\right], \quad Q_{\bar{r}}=\left[\begin{array}{ccc}
Q_{\bar{r} 11} & \cdots & Q_{\bar{r} 1 \alpha} \\
\vdots & \ddots & \vdots \\
Q_{\bar{r} \alpha 1} & \cdots & Q_{\bar{r} \alpha \alpha}
\end{array}\right]
$$

The first relationship in (38) leads that

$$
\begin{cases}J_{i}=Q_{p i i}+Q_{\bar{r} i i}, & i=1,2, \cdots, \alpha \\ Q_{r i k}=-Q_{\bar{r} i k}, & i, k=1,2 \cdots, \alpha, i \neq k\end{cases}
$$

Based on the blockwise expressions of $Q_{r}$ and $Q_{\bar{r}}$, it is trivial to see by (39) that

$$
\left\{\begin{array}{l}
{\left[Q_{r} Q_{\bar{r}}\right]_{i k}=\sum_{l=1}^{\alpha} Q_{r i l} Q_{\bar{r} l k}=-\sum_{l=1, l \neq i, l \neq k}^{\alpha} Q_{r i l} Q_{r l k}+Q_{r i k} Q_{\bar{r} k k}-Q_{r i i} Q_{r i k}} \\
{\left[Q_{r} Q_{\bar{r}}\right]_{i k}=\sum_{l=1}^{\alpha} Q_{\bar{r} i l} Q_{r l k}=-\sum_{l=1, l \neq i, l \neq k}^{\alpha} Q_{r i l} Q_{r l k}+Q_{\bar{r} i i} Q_{r i k}-Q_{r i k} Q_{r k k}}
\end{array}\right.
$$

which, together with the fact of $\left[Q_{r} Q_{\bar{r}}\right]_{i k}=\left[Q_{\bar{r}} Q_{r}\right]_{i k}$ and (39), implies that

$$
Q_{r i k} J_{k}=J_{i} Q_{r i k}, \quad \forall i, k=1,2, \cdots, \alpha
$$

By the eigevalue assumption on $Q, \lambda\left(J_{k}\right)-\lambda\left(J_{i}\right) \neq 0$ whenever $i \neq k$. Linear algebraic equations theory and Kronecker products, say Theorem 5.2.2 of [17], tell that for any $i \neq k, Q_{r i k}=0$ is a unique solution to (40). Interpreting this in light of (39), we see that $Q_{r}$ and $Q_{\bar{r}}$ are blockwisely diagonal, and their diagonal blocks are in accordance with those of $Q$ in dimension.

Finally, we see by Theorem 14 that $Q_{r}$ and $Q$ are also commutative; i.e., $Q Q_{r}=Q_{r} Q$. This, together with the facts that $Q$ and $Q_{r}$ are blockwisely diagonal, implies that a Jordan block in $Q$ must be commutative with a corresponding block in $Q_{r}$. Therefore, it causes no loss of generality if we assume that $Q$ has only one Jordan block corresponding to an eigenvalue $\lambda \in \mathcal{C}_{f / k}$.

To show that some contradictions will be derived eventually under the reducibleness assumption, we consider two cases according to the eigenvalue multiplicity of $Q$.

Case (a). $Q$ is diagonal. We can write $Q_{\bar{r}}=\operatorname{diag}[\lambda, \cdots, \lambda]-\left[q_{i k}\right]=: Q-Q_{r}$ where $Q$ and $Q_{r}$ are square. Hence, $\lambda_{i}\left(Q_{\bar{r}}\right)=\lambda-\lambda_{i}\left(Q_{r}\right)$. Since $Q_{r}$ has at least one eigenvalue $j \omega_{k h} \mu_{i} \neq 0$, thus at least one eigenvalue of $Q_{\bar{r}}$ does not belong to $\mathcal{C}_{f / k}$. This is contradictory to (38).

Case (b). $Q$ is a Jordan block with multiplicity two or larger. That is, we can write

$$
Q_{\bar{r}}=\underbrace{\left[\begin{array}{cccc}
\lambda & 1 & \cdots & 0 \\
0 & \lambda & \cdots & 0 \\
\vdots & \vdots & \ddots & \vdots \\
0 & 0 & \cdots & \lambda
\end{array}\right]}_{Q}-\underbrace{\left[\begin{array}{cccc}
q_{11} & q_{12} & \cdots & q_{1 n} \\
q_{21} & q_{22} & \cdots & q_{2 n} \\
\vdots & \vdots & \ddots & \vdots \\
q_{n 1} & q_{n 2} & \cdots & q_{n n}
\end{array}\right]}_{Q_{r}}
$$


Now let us re-write $Q_{r}$ as follows.

$$
\begin{aligned}
Q_{r} & =\left[\begin{array}{cccc}
0 & 0 & \cdots & 0 \\
q_{21} & 0 & \cdots & 0 \\
\vdots & \vdots & \ddots & \vdots \\
q_{n 1} & q_{n 2} & \cdots & 0
\end{array}\right]+\left[\begin{array}{cccc}
q_{11} & q_{12} & \cdots & q_{1 n} \\
0 & q_{22} & \cdots & q_{2 n} \\
\vdots & \vdots & \ddots & \vdots \\
0 & 0 & \cdots & q_{n n}
\end{array}\right] \\
& =: \quad Q_{l r}+Q_{u r}
\end{aligned}
$$

where $Q_{l r}$ and $Q_{u r}$ have the obvious definitions.

Furthermore, it follows from Theorem 14 that $Q_{r} Q=Q Q_{r}$. Interpreting this commutativity in terms of $Q, Q_{u p}$ and $Q_{l p}$, we are led that $Q Q_{u r}+Q Q_{l r}=Q_{u r} Q+Q_{l r} Q$. Clearly, $Q Q_{u r}$ and $Q_{u r} Q$ are upper trianglar matrices, whereas $Q Q_{l r}$ and $Q_{l r} Q$ are lower trianglar ones. Now we concentrate our attention only on the entries locating at the $(n, n)$-position and the lower trianglar portions of matrices in $Q Q_{u r}+Q Q_{l r}=Q_{u r} Q+Q_{l r} Q$. Computing $Q Q_{l r}$ and $Q_{l r} Q$ and comparing the two sides of $Q Q_{u r}+Q Q_{l r}=Q_{u r} Q+Q_{l r} Q$, we obtain

$$
\begin{aligned}
& \left\{\begin{array}{l}
\lambda q_{21}+q_{31}=\lambda q_{21} \\
\cdots \\
\lambda q_{n-1,1}+q_{n 1}=\lambda q_{n-1,1} \\
\lambda q_{n 1}=\lambda q_{n 1}
\end{array},\left\{\begin{array}{l}
\lambda q_{32}+q_{42}=q_{31}+\lambda q_{32} \\
\ldots \\
\lambda q_{n-1,2}+q_{n 2}=q_{n-1,1}+\lambda q_{n-1,2} \\
\lambda q_{n 2}=q_{n 1}+\lambda q_{n 2}
\end{array}, \cdots,\right.\right. \\
& \left\{\begin{array}{l}
\lambda q_{n, n-1}=q_{n, n-2}+\lambda q_{n, n-1} \\
\lambda q_{n n}=q_{n, n-1}+\lambda q_{n n}
\end{array}\right.
\end{aligned}
$$

which produce us that

$$
\left\{\begin{array}{l}
q_{31}=0 \\
\cdots \\
q_{n-1}=0 \\
q_{n 1}=0
\end{array}, \quad\left\{\begin{array}{l}
q_{42}=0 \\
\cdots \\
q_{n-1,2}=0 \\
q_{n 2}=0
\end{array}, \cdots, \quad\left\{\begin{array}{l}
q_{n, n-2}=0 \\
q_{n, n-1}=0
\end{array}\right.\right.\right.
$$

The above arguments indicate that (41) can be re-written as follows.

$$
Q_{\bar{r}}=\operatorname{diag}\left[\lambda-q_{11}, \cdots, \lambda-q_{n n}\right]+[*]
$$

where $[*]$ is an upper triangular matrix with zero diagonal entries. Taking into account the fact that all the eigenvalues of $Q_{r}$ can be written as $j \omega_{k h} \mu_{i}$, the triangular expression of $Q_{r}$ means that $q_{i i}=j \omega_{k h} \mu_{i}$. Since at least one eigenvalue $j \omega_{k h} \mu_{i}$ is nonzero, we see by (42) that at least one eigenvalue of $Q_{\bar{r}}$ is not in $\mathcal{C}_{f / k}$. This is again contradictory to $\lambda\left(Q_{\bar{r}}\right) \subset \mathcal{C}_{f / k}$ in (38).

The contradictions in the above two cases show that the reducing factor $e^{Q_{r} t}$ satifying (38) does not exist. Or equivalently, one can say that (38) holds only if $Q_{r}=0$.

\section{References}

[1] F. M. Arscott, Periodic Differential Equations: An Introduction to Mathieu, Lamé, and Allied Functions, Pergamon Press, Oxford, 1964.

[2] A. Böttcher and B. Silbermann, Analysis of Toeplitz operators, Springer-Verlag, Berlin, 1990.

[3] R. V. Churchill, Fourier Series and Boundary Value Problems, McGraw-Hill, 2nd Ed, 1963. 
[4] E. A. Coddington and N. Levinson, Theory of Ordinary Differential Equations, McGraw-Hill, New York, 1955.

[5] W. J. Culver, "On the existence and uniqueness of the real logarithm of a matrix," Proc. Amer. Math. Soc., vol. 17, no. 5, pp. 1146-1151, 1966.

[6] J. Dugundji and J. H. Wendelll, "Some analysis methods for rotating systems with periodic coefficients," AIAA J., vol. 21, pp. 890-897, 1983.

[7] M. S. P. Eastham, The spectral theory of periodic differential equations, Scottish Academic Press, Edinburgh, 1973.

[8] M. Farkas, Periodic Motions, Springer-Verlag, 1994.

[9] G. Floquet, "Sur les équations linéaires à coefficients périodiques," Ann. Sci. "École Normale Sup'erieure, Ser. 2 (Suppl. 12), pp. 47-88, 1883.

[10] H. Fukawa, Mathematics in Control and Vibration, Corona, Tokyo, 1974 (in Japanese).

[11] M. Grimble, Industrial Control Systems Design, Wiley, 2001.

[12] A. Halanay, Differential Equations: Stability, Oscillations, Time Lags, Academic Press, 1966.

[13] J. K. Hale, Ordinary Differential Equations, Wiley-Interscience, New York, 1969.

[14] P. Hartman, Ordinary Differential Equations, 2nd Ed., Birkhäuser, Boston, 1982.

[15] R. A. Horn and C. A. Johnson, Matrix Analysis, Cambridge University Press, Cambridge, 1985.

[16] P. Kuchment, Floquet Theory for Partial Differential Equations, Birkhäuser, Basel, 1993.

[17] P. Lancaster and L. Rodman, Algebraic Riccati Equations, Clarendon Press, Oxford, 1995.

[18] S. Lang, Complex Analysis, Springer-Verlag, New York, 1999.

[19] D. L. Lukes, Differential Equations: Classical to Controlled, Academic Press, 1982.

[20] P. Montagnier, C. C. Paige and R. J. Spiteri, "Real Floquet factors of linear time-periodic systems," Systems \& Control Lett., vol. 50, pp. 251-262, 2003.

[21] P. Montagnier and R. J. Spiteri, "A Gramian-based controller for linear periodic systems," IEEE Trans. Automat. Contr., vol. 49, no. 8, pp. 1380-1385, 2004.

[22] P. Montagnier, R. J. Spiteri and J. Angeles, "The control of linear time-periodic systems using Floquet-Lyapunov theory," Int. J. Control, vol. 77, no. 5, pp. 472-490, 2004.

[23] T. Myint-U, Ordinary Differential Equations, North-Holland, New-York, 1978.

[24] M. Pavella and P. G. Murthy, Transient Stability of Power System-Theory and Practice. John Wiley \& Sons, 1994.

[25] J. A. Richards, Analysis of Periodically Time-Varying Systems, Springer-Verlag, 1983. 
[26] W. Rudin, Real and Complex Analysis, McGraw-Hill Book Company, New York, 3rd, 1987.

[27] N. M. Wereley, Analysis and Control of Linear Periodically Time Varying Systems, Ph. D Thesis, Dept. of Aeronautics and Astronautics, M.I.T., 1990.

[28] V. A. Yakubovich and V. M. Starzhinskii, Linear Differential Equations wiht Periodic Coefficients, vol. 1, John Wiley \& Sons, New York, 1975.

[29] R. Wadanabe, H. Miyazaki and S. Endo, Complex Analysis, Peifu-Kan, Tokyo, 1979 (in Japanese).

[30] J. Zhou and T. Hagiwara, "Existence conditions and properties of frequency response operators of continuous-time periodic systems," SIAM Journal on Control and Optimization, vol. 40, no.6, pp. 1867-1887, 2002.

[31] J. Zhou, T. Hagiwara and M. Araki, "Stability analysis of continuous-time periodic systems via the harmonic analysis," IEEE Trans. Automatic Control, vol. 47, no. 2, pp. 292-298, 2002.

[32] J. Zhou, T. Hagiwara and M. Araki, "Spectral characteristics and eigenvalues computations of the harmonic state operators in continuous-time periodic systems," Systems \& Control Lett., vol. 53, no. 2, pp. 141-155, 2004.

[33] J. Zhou and T. Hagiwara, "2-regularized Nyquist stability criterion in linear continuous-time periodic systems," SIAM Journal on Control and Optimization, vol. 44, no. 2, pp. 618-645, 2005.

[34] J. Zhou, "A harmonic framework for controllability in linear continuous-time periodic systems," SIAM Journal on Control and Optimization, vol. 46, no. 2, pp. 630-654, 2007. 